\title{
ON THE EARLY-TIME EXCESS EMISSION IN HYDROGEN-POOR SUPERLUMINOUS SUPERNOVAE
}

\author{
Paul M. Vreeswijk ${ }^{1,22}$, Giorgos Leloudas ${ }^{1,2}$, Avishay Gal-Yam ${ }^{1}$, Annalisa De Cia ${ }^{1,3}$, Daniel A. Perley ${ }^{2,4}$, \\ Robert M. Quimby ${ }^{5,6}$, Roni Waldman ${ }^{1,7}$, Mark Sullivan $^{8}$, Lin Yan ${ }^{9}$, Eran O. Ofek ${ }^{1}$, Christoffer Fremling ${ }^{10}$, \\ Francesco Taddia $^{10}$, Jesper Sollerman ${ }^{10}$, Stefano VAlenti ${ }^{11}$, IaIr ArCavi ${ }^{12,13,23}$, D. Andrew Howell ${ }^{12,13}$, \\ Alexei V. Filippenko ${ }^{14}$, S. Bradley Cenko ${ }^{15,16}$, Ofer Yaron ${ }^{1}$, Mansi M. Kasliwal ${ }^{4}$, Yi CaO ${ }^{4}$, Sagi Ben-Ami ${ }^{1,17}$, \\ Assaf Horesh ${ }^{1}$, Adam Rubin ${ }^{1}$, Ragnhild Lunnan ${ }^{4}$, Peter E. Nugent ${ }^{14,18}$, Russ Laher ${ }^{19}$, UmaA D. Rebbapragada ${ }^{20}$, \\ PrzemysŁaw Woźniak ${ }^{21}$, and Shrinivas R. Kulkarni ${ }^{4}$ \\ ${ }^{1}$ Department of Particle Physics and Astrophysics, Weizmann Institute of Science, Rehovot 7610001, Israel; paul.vreeswijk@weizmann.ac.il \\ ${ }^{2}$ Dark Cosmology Centre, Niels Bohr Institute, University of Copenhagen, Juliane Maries Vej 30, DK-2100 København Ø, Denmark \\ ${ }^{3}$ European Southern Observatory, Karl-Schwarzschild-Strasse 2, D-85748 Garching bei München, Germany \\ ${ }^{4}$ Cahill Center for Astrophysics, California Institute of Technology, Pasadena, CA 91125, USA \\ ${ }^{5}$ Department of Astronomy, San Diego State University, San Diego, CA 92182, USA \\ ${ }^{6}$ Kavli IPMU (WPI), UTIAS, The University of Tokyo, Kashiwa, Chiba 277-8583, Japan \\ ${ }^{7}$ Racah Institute of Physics, The Hebrew University, Jerusalem 91904, Israel \\ ${ }^{8}$ School of Physics and Astronomy, University of Southampton, Southampton SO17 1BJ, UK \\ ${ }^{9}$ Infrared Processing and Analysis Center, California Institute of Technology, Pasadena, CA 91125, USA \\ ${ }^{10}$ The Oskar Klein Centre, Department of Astronomy, Stockholm University, AlbaNova, SE-10691 Stockholm, Sweden \\ ${ }^{11}$ Department of Physics, University of California, Davis, CA 95616, USA \\ ${ }^{12}$ Department of Physics, University of California, Santa Barbara, CA 93106, USA \\ ${ }^{13}$ Las Cumbres Observatory Global Telescope, 6740 Cortona Drive, Suite 102, Goleta, CA 93111, USA \\ ${ }^{14}$ Department of Astronomy, University of California, Berkeley, CA 94720-3411, USA \\ 15 Astrophysics Science Division, NASA Goddard Space Flight Center, Mail Code 661, Greenbelt, MD 20771, USA \\ ${ }^{16}$ Joint Space-Science Institute, University of Maryland, College Park, MD 20742, USA \\ ${ }^{17}$ Smithsonian Astrophysical Observatory, Harvard-Smithsonian Center for Astrophysics, 60 Garden Street, Cambridge, MA 02138, USA \\ ${ }^{18}$ Computational Cosmology Center, Lawrence Berkeley National Laboratory, 1 Cyclotron Road, Berkeley, CA 94720, USA \\ ${ }^{19}$ Spitzer Science Center, MS 314-6, California Institute of Technology, Pasadena, CA 91125, USA \\ ${ }^{20}$ Jet Propulsion Laboratory, California Institute of Technology, Pasadena, CA 91109, USA \\ ${ }^{21}$ Los Alamos National Laboratory, MS D436, Los Alamos, NM 87545, USA \\ Received 2016 September 21; revised 2016 November 29; accepted 2016 November 29; published 2017 January 18
}

\begin{abstract}
We present the light curves of the hydrogen-poor superluminous supernovae (SLSNe I) PTF 12dam and iPTF 13dcc, discovered by the (intermediate) Palomar Transient Factory. Both show excess emission at early times and a slowly declining light curve at late times. The early bump in PTF 12dam is very similar in duration ( $\sim 10$ days) and brightness relative to the main peak (2-3 mag fainter) compared to that observed in other SLSNe I. In contrast, the long-duration ( $>30$ days) early excess emission in iPTF 13dcc, whose brightness competes with that of the main peak, appears to be of a different nature. We construct bolometric light curves for both targets, and fit a variety of light-curve models to both the early bump and main peak in an attempt to understand the nature of these explosions. Even though the slope of the late-time decline in the light curves of both SLSNe is suggestively close to that expected from the radioactive decay of ${ }^{56} \mathrm{Ni}$ and ${ }^{56} \mathrm{Co}$, the amount of nickel required to power the full light curves is too large considering the estimated ejecta mass. The magnetar model including an increasing escape fraction provides a reasonable description of the PTF 12dam observations. However, neither the basic nor the double-peaked magnetar model is capable of reproducing the light curve of iPTF 13dcc. A model combining a shock breakout in an extended envelope with late-time magnetar energy injection provides a reasonable fit to the iPTF 13dcc observations. Finally, we find that the light curves of both PTF 12dam and iPTF 13dcc can be adequately fit with the model involving interaction with the circumstellar medium.
\end{abstract}

Key words: supernovae: general - supernovae: individual (PTF 12dam, iPTF 13dcc)

Supporting material: data behind figure, machine-readable tables

\section{INTRODUCTION}

Supernovae that reach an absolute magnitude brighter than the (arbitrary) limit of $M=-21$ are labeled superluminous (Gal-Yam 2012). Even though they are very rare, several tens of them have been discovered over the past decade thanks to the ever-increasing survey speed of optical telescopes. They are observationally separated into two classes based on the detection of hydrogen in their spectra, similar to classical

\footnotetext{
22 Benoziyo Fellow.

23 Einstein Fellow.
}

supernovae (see Filippenko 1997): hydrogen-rich Type II superluminous supernovae (SLSNe) show clear Balmer features (e.g., Ofek et al. 2007; Smith et al. 2007), while the hydrogen-poor Type I SLSNe do not (Gal-Yam 2012). The latter commonly exhibit a distinct $\mathrm{W}$-shaped feature identified as O II at rest-frame wavelengths $4000-4500 \AA$ (Quimby et al. 2011; Mazzali et al. 2016). At late times, the spectra of these SLSNe I evolve to appear like those of normal Type Ic SNe (Pastorello et al. 2010), leading many authors to refer to this class as SLSN Ic. Sometimes this Type I/II distinction is not so obvious; for example, spectra of CSS121015:004244+132827 (hereafter referred to as CSS 121015) have similarities to both 
Type II and Type I objects (Benetti et al. 2014), while iPTF 13ehe, classified as Type I, shows the emergence of broad $\mathrm{H} \alpha$ emission at late times (Yan et al. 2015; see also Moriya et al. 2015; Wang et al. 2016).

There is some evidence that the energy source powering the hydrogen-rich SLSNe is interaction of the SN ejecta with optically thick material at a large distance $\left(\sim 10^{15} \mathrm{~cm}\right)$, because they typically reveal Balmer emission lines indicative of interaction with a hydrogen-rich circumstellar medium (CSM; e.g., Chevalier \& Fransson 1994; Chugai \& Danziger 1994; Ofek et al. 2014). Because of the similarity with normal SNe of Type IIn, this class is also referred to as SLSNe IIn. However, some SLSNe II do not exhibit narrow emission lines, while they are of Type II because they reveal broad hydrogen features during the photospheric phase (Inserra et al. 2016; see also Moriya \& Tominaga 2012).

The energy source of the hydrogen-poor SLSNe is still under debate, with the most promising candidates being (1) additional energy input from a central engine, such as a spinning-down magnetar (e.g., Kasen \& Bildsten 2010; Woosley 2010; Inserra et al. 2013) or an accreting black hole (Dexter \& Kasen 2013); (2) interaction of the ejecta with a hydrogen-poor shell expelled by the progenitor star some time before the explosion (Blinnikov \& Sorokina 2010; Chevalier \& Irwin 2011); and (3) the radioactive decay of a large amount of nickel produced in the explosion, potentially due to conditions of pair instability (see Gal-Yam et al. 2009) though this is still being debated (Moriya et al. 2010; Young et al. 2010; Nicholl et al. 2013). The light curves of some hydrogen-poor SLSNe, such as the two PTF sources presented in this paper, decay very slowly at late times, with a slope similar to that expected from the decay of radioactive nickel and cobalt. These SLSNe are part of the hydrogen-poor class, but are sometimes referred to as Type $\mathrm{R}$ ("radioactive"; see Gal-Yam 2012).

The host galaxies of SLSNe are found to be irregular, compact, low-mass galaxies with high specific star formation rates (Neill et al. 2011; Lunnan et al. 2014, 2015; Leloudas et al. 2015). A comprehensive study of 32 host galaxies of all PTF-discovered SLSNe (until the end of 2012) found hydrogen-poor SLSNe to have a preference for environments in hosts with an upper bound on metallicity of about half solar, while the hydrogen-rich SLSNe do not show such a preference (Perley et al. 2016). A very similar conclusion was reached independently by Chen et al. (2016). In emission, the galaxies hosting SLSNe have broadly similar characteristics to the hosts of gamma-ray bursts (GRBs). Leloudas et al. (2015) find the host-galaxy emission-line strengths of SLSNe I at redshift $z<1$ to be significantly stronger than in GRB hosts, but Japelj et al. (2016) do not confirm this result over the range $0.3<z<0.7$. In absorption, the environments of SLSNe I appear to be significantly poorer in their neutral gas content, as traced by $\mathrm{Mg} \mathrm{I}$ and $\mathrm{Mg}$ II, than those of GRBs (Vreeswijk et al. 2014).

Focusing on the hydrogen-poor class, several of these have shown evidence for an early-time "bump" or excess emission in the light curve before the onset of the main peak. Examples are SN 2006oz (Leloudas et al. 2012), LSQ 14bdq (Nicholl et al. 2015), and DES 14X3taz (Smith et al. 2016). In fact, Nicholl \& Smartt (2016) suggest that early bumps such as the ones above may be ubiquitous in hydrogen-poor SLSNe. Early bumps have also been observed in normal stripped-envelope $\mathrm{SNe}$, and recently in a normal $\mathrm{SN}$ Ic from a massive progenitor (Taddia et al. 2016). To date, such early excess emission has not been reported for any hydrogen-rich SLSN II. This early excess emission is of particular interest, because it may provide a clue to what is powering these explosions.

In the case of SN 2006oz, Leloudas et al. (2012) propose that the precursor bolometric plateau might be related to a recombination wave in an H-poor CSM. The study by Moriya \& Maeda (2012) has shown that a dip in the light curve is naturally expected when shock breakout occurs within a dense CSM. Nicholl et al. (2015) propose that the initial peak in LSQ 14bdq may arise from the post-shock cooling of extended stellar material, while reheating by a central engine is driving the main peak. The high kinetic energy inferred from fitting the model of Rabinak \& Waxman (2011) to the initial peak $\left(E_{\mathrm{k}} \sim 2 \times 10^{52} \mathrm{erg}\right)$ of LSQ 14 bdq may favor a black hole accretion engine (Dexter \& Kasen 2013) rather than a magnetar. The early-time excess emission in the case of DES $14 X 3$ taz shows rapid cooling from 22,000 to $8000 \mathrm{~K}$ over the course of 15 rest-frame days. The authors find that a shockcooling model of CSM at a distance of $\sim 400 R_{\odot}$, followed by a magnetar causing the main peak of the light curve, can adequately explain the entire light curve.

In this paper, we present two SLSNe I discovered by the (intermediate) Palomar Transient Factory (Rau et al. 2009; Law et al. 2009) that also show evidence for early excess emission: PTF 12dam and iPTF 13dcc. The modest bump in PTF 12dam is very similar in duration and brightness relative to the main peak to the cases discussed above. However, the long-duration early excess emission in iPTF $13 \mathrm{dcc}$, whose brightness competes with that of the main peak, appears to be of a different nature.

This paper is organized as follows. In Section 2 we present the photometric observations that we obtained for PTF 12dam and iPTF 13dcc, as well as the spectroscopic sequence of iPTF 13 dcc. We construct the bolometric luminosity evolutions in Section 3, which we confront with models in Section 4. The models (see Chatzopoulos et al. 2012, 2013; Piro 2015) assume different energy sources (radioactive decay, magnetar heating, and CSM interaction) and predict the ensuing light curve based on a number of parameters; we infer estimates of the best-fit values by fitting the semianalytical models to the bolometric light curves. We discuss our results and briefly conclude in Section 5.

Unless noted otherwise, the uncertainties listed in this paper are at the $1 \sigma$ confidence level. We adopt the cosmological parameters as derived by the Planck collaboration in 2015 $\left(H_{0}=68 \mathrm{~km} \mathrm{~s}^{-1} \mathrm{Mpc}^{-1}, \Omega_{\mathrm{m}}=0.31, \Omega_{\Lambda}=0.69\right.$; Planck Collaboration et al. 2016).

\section{IDENTIFICATION, OBSERVATIONS, AND DATA REDUCTION}

\subsection{PTF 12 dam}

PTF 12dam was flagged as a transient source as part of the regular PTF operations on 2012 April 17 (UTC dates are used throughout this paper); it was first detected on April 10. The source is located at $\alpha=14^{\mathrm{h}} 24^{\mathrm{m}} 46^{\mathrm{s}} .20, \delta=+46^{\circ} 13^{\prime} 48^{\prime \prime} \cdot 3$ (J2000.0), with an uncertainty of 0 !' 1 . At this location the Galactic extinction is estimated to be low, $A_{V}=0.033 \mathrm{mag}$ (Schlafly \& Finkbeiner 2011; see also Cardelli et al. 1989). Spectroscopic follow-up observations were performed with the Kast Spectrograph (Miller \& Stone 1993) at the Lick 3 m Shane 
telescope, and the Low Resolution Imaging Spectrograph (LRIS; Oke et al. 1995) at the Keck-I $10 \mathrm{~m}$ telescope (on Mauna Kea, Hawaii) on 2012 May 20, 21, and 22, showing PTF 12dam to be an SLSN I at $z=0.107$ (Quimby et al. 2012). The full spectroscopic sequence of PTF 12dam will be presented by R. M. Quimby et al. (2016, in preparation).

PTF 12dam was imaged with the Palomar Oschin 48 inch (P48) (i)PTF survey telescope equipped with a $12 \mathrm{k} \times 8 \mathrm{k}$ CCD mosaic camera (Rahmer et al. 2008) in the Mould $R$ filter, the Palomar 60 inch (P60) and CCD camera (Cenko et al. 2006) in Johnson $B$ and Sloan Digital Sky Survey (SDSS) gri, the Las Cumbres Observatory Global Telescope Network (LCOGT; Brown et al. 2013) in SDSS $r$, and LRIS mounted on the $10 \mathrm{~m}$ Keck-I telescope in $R_{s}$. Post-peak and late-time imaging shows PTF 12dam to have a slowly declining light curve, i.e., of Type $\mathrm{R}$ following the SLSN classification suggested by GalYam (2012).

PTF 12dam, relatively nearby at $z=0.107$, has already received considerable interest in the literature. Nicholl et al. (2013) use its light curve to argue against the claim that SN 2007bi, also an SLSN-R with a similar late-time light curve to PTF 12dam, is produced by a pair-instability explosion (GalYam et al. 2009). Chen et al. (2015) study both the late-time SN decay and the host galaxy of PTF 12dam. They find that its light curve can be fit with a magnetar model (see Kasen \& Bildsten 2010; Woosley 2010) if an escape fraction, increasing with time, of the magnetar energy input is considered (see also Wang et al. 2015). An even better fit is reached with a model involving the interaction of the ejecta with dense CSM. Using radiation-hydrodynamics calculations, Baklanov et al. (2015) show that PTF12dam and similar SLSNe can be explained without a magnetar in a model with a radiative shock in a dense circumstellar envelope. The host galaxy of PTF 12dam is a compact dwarf galaxy of low mass $\left(3 \times 10^{8} M_{\odot}\right)$ and low metallicity $(12+\log [\mathrm{O} / \mathrm{H}]=8.05 \pm 0.09)$ with a high starformation rate $\left(5 M_{\odot} \mathrm{yr}^{-1}\right.$; Chen et al. 2015). Thöne et al. (2015) report on long-slit spectroscopy of the host to infer the presence of a very young stellar population (even down to about $3 \mathrm{Myr}$ ).

\section{2. iPTF $13 d c c$}

iPTF 13dcc has not had any exposure in the literature yet. It was flagged as a transient source on 2013 August 29, which is the day that it was also first detected, with a magnitude of $r \approx 19.7$. It was initially slowly fading, but it surprisingly rebrightened by almost a magnitude, tens of days after discovery. iPTF had not observed this field for almost a year before this date (2012 September 28). The Catalina Sky Survey (CSS; Drake et al. 2009) monitored this field in early 2013 January, ${ }^{24}$ but there are no additional detections or useful limits during the months preceding the date of discovery. The images of the iPTF 13dcc field from the 2010 and 2012 PTF observing campaigns only show upper limits at the iPTF 13 dcc location. Its sky coordinates are $\alpha=2^{\mathrm{h}} 57^{\mathrm{m}} 02^{\mathrm{s}} .50, \delta=-00^{\circ} 18^{\prime} 44^{\prime \prime} 0$ (J2000.0), with an uncertainty of 0 !' 1 . It was independently discovered by the Catalina Real Time Survey (CRTS) on 2013 September 12 and given the name CSS130912:025702 -001844 (Drake et al. 2013). At this location the Galactic extinction is estimated to be moderate, $A_{V}=0.18 \mathrm{mag}$ (Cardelli et al. 1989; Schlafly \& Finkbeiner 2011).

\footnotetext{
${ }^{24}$ See http://nesssi.cacr.caltech.edu/DataRelease.
}

Spectroscopic follow-up observations were performed with the Double Spectrograph (DBSP) at the Palomar 200 inch (P200), LRIS at Keck-I, and the Inamori-Magellan Areal Camera \& Spectrograph (IMACS) at the Magellan Baade telescope, showing iPTF 13dcc to be an SLSN at $z=0.4305$.

iPTF 13dcc was imaged with the P48 Oschin (i)PTF survey telescope in the Mould $R$ filter, the P60 in SDSS gri, the $4.3 \mathrm{~m}$ Discovery Channel Telescope (DCT, at Lowell Observatory, Arizona) with the Large Monolithic Imager (LMI) in SDSS ri, and finally with the Hubble Space Telescope (HST) Advanced Camera for Surveys (ACS) Wide-Field Camera using filter F625W (under program GO-13858; P.I. A. De Cia).

\subsection{Data Reduction}

All PTF 12dam and iPTF 13dcc images were reduced in a standard fashion; for the P48 images this was done using the IPAC pipeline (Laher et al. 2014). Image-subtraction pointspread function (PSF) photometry is performed on all P48 and P60 images using a custom routine written by one of us (M.S.). This pipeline is described by Firth et al. (2015); it constructs deep reference images - from either before the SN explosion or after the SN has faded-and astrometrically aligns the images using the Automated Astrometry described by Hogg et al. (2008) and the Naval Observatory Merged Astrometric Data set (NOMAD; Zacharias et al. 2004). The image PSFs are matched in order to perform image subtraction, and PSF photometry is extracted from the difference image at the SN location. The fluxes are calibrated against the SDSS Data Release 10 (Ahn et al. 2014) when available, and otherwise using the photometric catalog of Ofek et al. (2012). The LCOGT data have been reduced using a custom pipeline developed by one of us (S.V.). This pipeline is described in the Appendix of Valenti et al. (2016), and employs standard procedures (PYRAF, DAOPHOT) in a PYTHON framework. Hostgalaxy flux was removed using image-subtraction technique HOTPANTS $^{25}$. PSF magnitudes were computed on the subtracted images and transformed to the standard SDSS filter system via observations of standard stars taken during clear nights. The logs of the photometric observations of PTF 12dam and iPTF 13dcc are presented in Tables 10 and 11, respectively.

The iPTF 13dcc spectra were reduced using standard pipelines and will be made digitally available via WISeREP (Yaron \& Gal-Yam 2012). The log of these spectroscopic observations is given in Table 1 .

\section{PHOTOMETRIC EVOLUTION}

\subsection{PTF 12dam}

Figure 1 shows the resulting light curve of PTF 12dam, combining data from our PTF campaign (filled circles, squares, and triangles for P48, P60, and LCOGT data, respectively) with measurements published in the literature (open circles) from Nicholl et al. (2013) and Chen et al. (2015), which include ultraviolet (UV) observations by Swift (Gehrels et al. 2004). All UV and optical filters are in the AB system (Oke \& Gunn 1983), while the near-infrared (NIR) filters $J H K$ are in the Vega system. The magnitudes shown have been corrected for the Galactic extinction along this sightline $\left(E_{B-V}=0.01 \mathrm{mag}\right.$;

\footnotetext{
${ }^{25}$ High Order Transform of PSF ANd Template Subtraction; http://www. astro.washington.edu/users/becker/v2.0/hotpants.html.
} 
Table 1

Log of Spectroscopic Observations of iPTF 13dcc

\begin{tabular}{|c|c|c|c|c|c|c|c|c|c|}
\hline UTC Date & Telescope & Instrument & $\begin{array}{l}\text { Exp. Time } \\
\text { (minutes) }\end{array}$ & Grating/Grism/Filter & $\begin{array}{l}\text { Slit Width } \\
(\operatorname{arcsec})\end{array}$ & $\begin{array}{c}\lambda \text { Coverage } \\
(\AA)\end{array}$ & $\begin{array}{l}\text { Res. }^{a} \\
(\AA)\end{array}$ & $\begin{array}{l}\text { I.Q. }^{\text {b }} \\
(\operatorname{arcsec})\end{array}$ & Airmass \\
\hline 2013 Nov 26 & P200 & DBSP & 20 & $600 / 4000,316 / 7500$ & 1.5 & $3400-10,400$ & 9.3 & 2.9 & 1.3 \\
\hline 2013 Dec 4 & Keck 1 & LRIS & 10 & $600 / 4000,400 / 8500$ & 1.0 & $3140-10,240$ & 5.8 & 1.2 & 1.4 \\
\hline 2013 Dec 31 & Magellan Baade & IMACS & 25 & Gra-300-4.3 & 0.9 & 3700-9700 & 6.1 & 0.9 & 1.3 \\
\hline 2014 Jan 6 & P200 & DBSP & 60 & $600 / 4000,316 / 7500$ & 1.5 & $3300-10,400$ & 8.5 & 1.8 & 1.6 \\
\hline
\end{tabular}

Notes.

${ }^{\mathrm{a}}$ The resolution of the spectra was determined from the width of the [O I] $\lambda 5577$ night-sky line.

b The image quality (I.Q.), or effective seeing, was measured directly from the width of the object's spatial profile around $6000 \AA$.

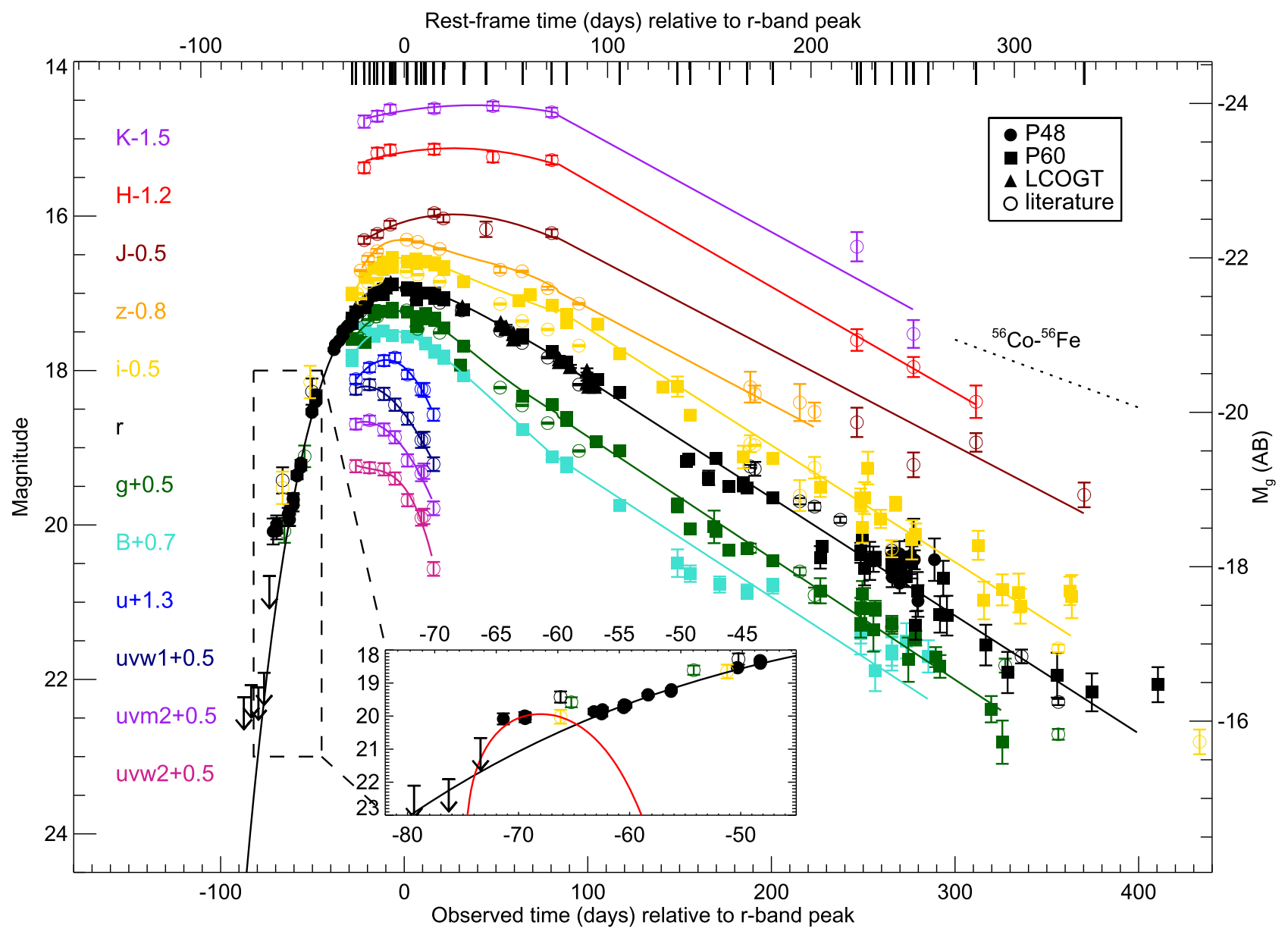

Figure 1. Light curves of PTF 12dam. Different colors and symbols denote different filters and telescopes, respectively. The solid lines show polynomial fits to the data points, where the early-time data (before $t_{\mathrm{obs}}=80$ days) are fit with a polynomial of order $2-5$, while the late-time data are fit with a straight line. The inset displays a zoom of the very early-time light curve, showing evidence for an initial plateau with a duration of about 5-10 days. The solid red line in the inset shows a fit to the early-time $r$-band light curve using the Piro (2015) model, explained in more detail in Section 4. The late-time light curve is not that different from the decay expected from full trapping of gamma-rays produced in the radioactive decay of ${ }^{56} \mathrm{Co}$ to ${ }^{56} \mathrm{Fe}$, as indicated by the dotted line. The short vertical lines at the top show the epochs at which we constructed spectral energy distributions to derive the bolometric light curve. The absolute $g$-band $\mathrm{AB}$ magnitude corresponding to the observed $r$-band light curve around peak is indicated on the right-hand axis.

Schlafly \& Finkbeiner 2011). We performed polynomial fits to the light curves (magnitudes versus linear time) with the order ranging from two (for filters with a sparsely sampled light curve, such as $J H K$ ) to five (for the well-sampled $r$-band data) at phases between -60 and +75 days (here and throughout the paper, phase refers to the time in days relative to the main peak in the $r$-band filter in the rest frame). For the filters with measurements beyond +75 days, the data are adequately described by a straight line-that is, an exponential decay of the flux in time. The combined early- and late-time fits are shown by the solid lines. For the filters Bgri these fits were performed only to PTF data. The observed late-time slopes are in the range $0.012-0.016 \mathrm{mag} \mathrm{day}^{-1}$ for all filters. The inset shows a close-up view of the early-time light curve, which includes a Piro (2015) model fit (solid red line) discussed in Section 4. 

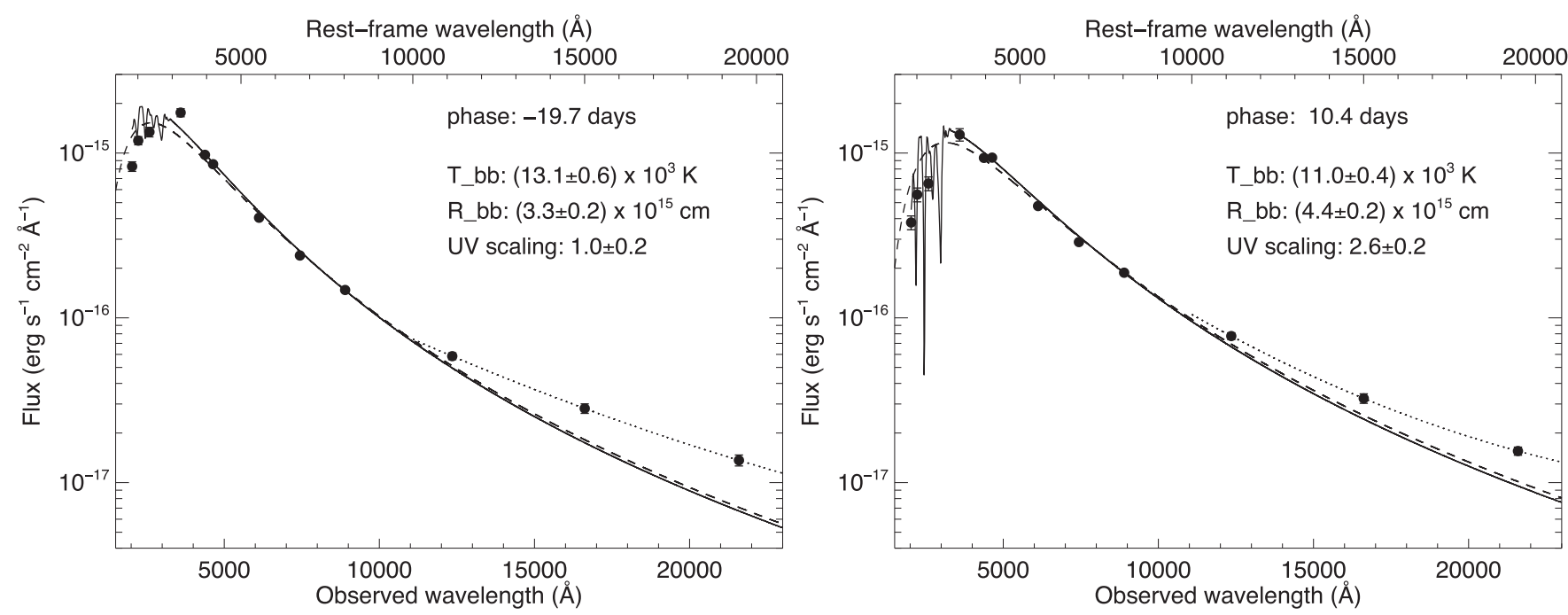

Figure 2. Two examples of Planck-function fits to the SEDs constructed from the photometry. The dashed lines show the best-fitting Planck function to the data, while the solid line depicts the best-fitting Planck function that has been modified below $3000 \AA$ using an observed near-UV spectrum taken with HST. Simply comparing the near-UV magnitudes (the three data points on the left) with the $u B g$ magnitudes suggests that the UV absorption is increasing with time. The dotted lines display a low-order polynomial fit to the NIR data, which is used to estimate the amount of NIR excess above the Planck fit. This NIR excess emission might be produced by reemission of photons absorbed in the near UV. The blackbody temperature, radius, and UV scaling factor derived from these fits are also listed (see the text for details).

The peak brightness in the observed $r$-band of $m_{r}=16.9$ mag is reached at a modified Julian date (MJD) of 56096.7, corresponding to UTC 2012 June 18.7. At this epoch, we determine a $K$-correction (see Hogg et al. 2002) from observed $r$ to rest-frame SDSS $g$ of -0.01 , resulting in an absolute $g$-band peak magnitude of $M_{g}$, peak $=-21.6$ (the distance modulus of PTF $12 \mathrm{dam}$ is $38.55 \mathrm{mag}$ ). The $K_{r g^{-}}$ correction is determined assuming that PTF 12dam is radiating like a blackbody, adopting the temperature evolution as inferred below (see Figure 3). The absolute $g$-band magnitude is plotted on the right-hand axis of Figure 1. We note that as the $K$-correction evolves in time $\left(K_{r g}\right.$ develops from +0.2 at a phase of -70 days to -1.0 at +334 days), the scale on this axis is valid only for the $r$-band light curve (in black) around peak magnitude. Our earliest detections of the SN, between -60 and -55 rest-frame days before the $r$-band peak, show evidence for significant emission in excess of that expected from extrapolation of the polynomial fit-see the inset in Figure 1. What appears to be a single data point in the light curve, at -70 days in the observer's frame, actually consists of three independent measurements, making the excess above the polynomial fit highly significant. This excess emission is clear only from the PTF data, and has not been inferred from imaging data sets published to date (e.g., Nicholl et al. 2013). The $r$-band limiting magnitudes shown have been determined by coadding all the images (typically three) taken during a single night.

In order to derive the bolometric light curve of PTF 12dam, we select a number of epochs at which we construct spectral energy distributions (SEDs). These correspond to the epochs at which observations were performed in the near-UV by Swift or the $B$ - or $K$-band filters; they are indicated as short solid lines at the top of Figure 1. We adopt the magnitude as estimated by the polynomial fits at a particular epoch, and the magnitude error from the measurement in the same filter closest in time to the SED epoch.

Two example SEDs, taken from epochs with phases -19.7 and +10.4 days, are shown in Figure 2. The overall shape of the SEDs for all epochs is very similar to that of a Planck function. We first fit Planck functions to the observed SEDs (which have been corrected for Galactic extinction) by converting the observed magnitude to flux in the rest frame at the central wavelengths of the filters. These fits provide an estimate of the blackbody effective temperature $\left(T_{\mathrm{bb}}\right)$ and radius $\left(R_{\mathrm{bb}}\right)$; they are shown by the dashed lines in the example SEDs in Figure 2. It is clear that both the near-UV and NIR parts of the SED are poorly fit.

We account for the additional UV absorption by utilizing the spectrum of PTF 12dam as observed by HST on 2012 May 26, at a phase of -20 days (R. M. Quimby et al. 2016, in preparation). This spectrum is well approximated by a Planck function redward of $3000 \AA$, while strong absorption features are present blueward of that. These near-UV features are at very similar wavelengths to the features observed in other SLSNe, such as SCP 06F6 (Barbary et al. 2009), SNLS 06D4eu (Howell et al. 2013), and iPTF 13ajg (Vreeswijk et al. 2014). We normalize the $H S T$ spectrum blueward of $3000 \AA$ (rest frame), so it can be used to modify the Planck function to be fit, as explained below. The flux below a restframe wavelength of $1800 \AA$ in this template spectrum is assumed to be zero, which is a fair assumption because the SLSNe with spectra down to this rest-frame wavelength show very low flux levels in that spectral region and the flux is rapidly declining toward the blue.

We again perform Planck-function fitting, but rather than converting magnitudes to rest-frame flux, we instead first construct a Planck spectrum at a distance of $10 \mathrm{pc}$ in the rest frame, based on the two fit parameters $T_{\mathrm{bb}}$ and $R_{\mathrm{bb}}$. This spectrum is then modified in the region $1800-3000 \AA$ with the normalized HST spectrum (in the rest frame), by simply multiplying the $H S T$ and the blackbody spectra over this wavelength region. Below $1800 \AA$ it is assumed to be zero, beyond $3000 \mathrm{~A}$ it is a pure blackbody. This template spectrum is then converted to the observer's frame (with the spectrum in units of $\mathrm{erg} \mathrm{s}^{-1} \mathrm{~cm}^{-2} \AA^{-1}$; the spectrum is multiplied by $\left(10 / d_{1, \mathrm{pc}}\right)^{2} /(1+z)$, where $d_{1, \mathrm{pc}}$ is the luminosity distance of PTF 12dam in parsecs), and magnitudes are extracted from this spectrum by performing synthetic photometry (using the filter 


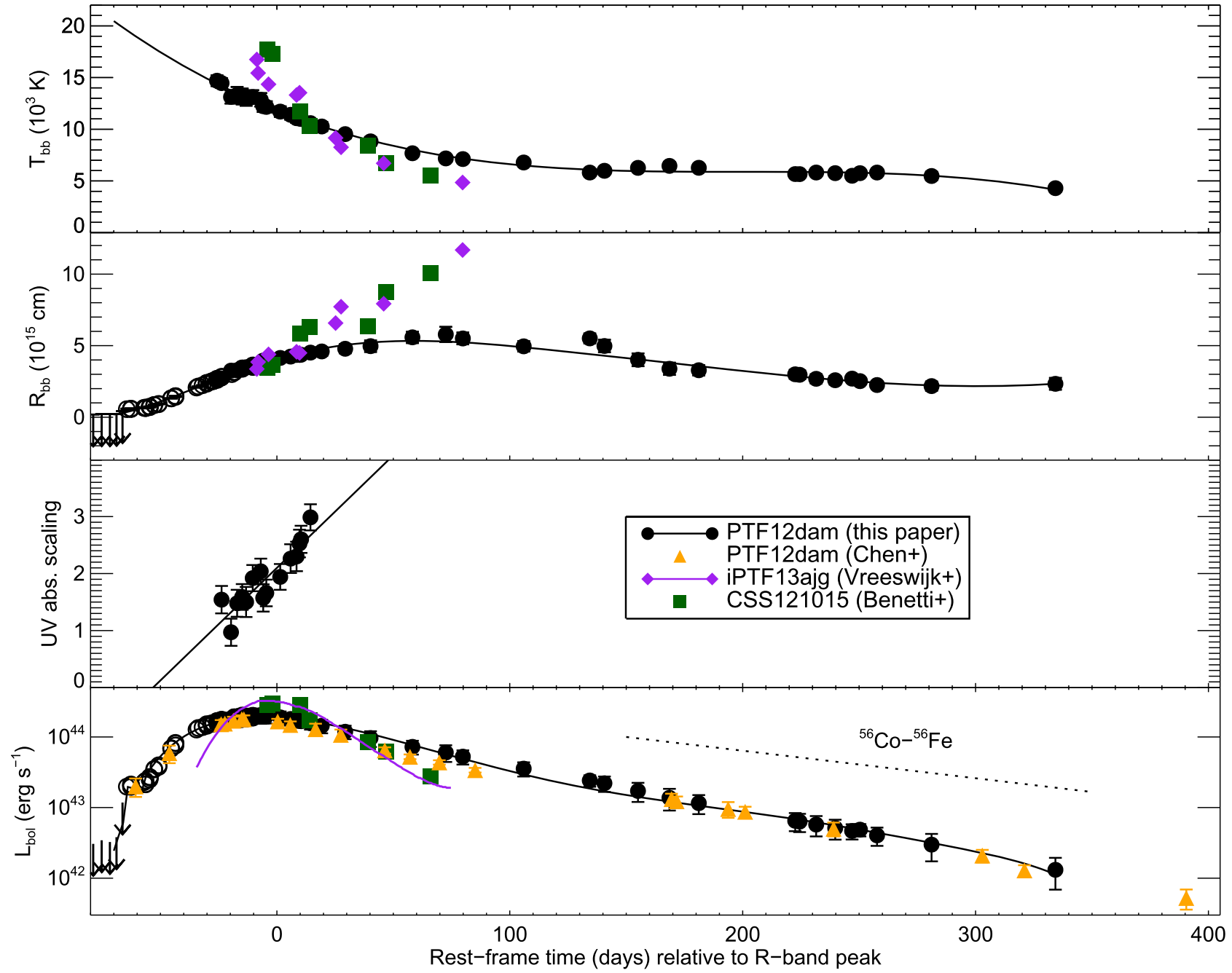

Figure 3. Time evolution of (from top to bottom) the blackbody effective temperature, blackbody radius, UV absorption strength scaling of observed HST spectrum, and bolometric luminosity of PTF 12dam compared to a few other SLSNe. The filled black circles correspond to the best-fit values inferred from the Planck-function fits to the SEDs constructed from the light curve, with fit parameters $T_{\mathrm{bb}}, R_{\mathrm{bb}}$, and UV absorption scale factor. The solid lines in the top three panels are polynomial fits to the best-fit values, and the solid black line in the lower panel is derived from these. The radii inferred from the early $r$-band data points alone (instead of the multifilter SEDs), adopting the temperature evolution shown in the top panel, are depicted by the open circles, as are the corresponding bolometric luminosity values. The filled circles in the bottom panel show the bolometric light curve of PTF 12dam inferred from the $T_{\mathrm{bb}}$ and $R_{\mathrm{bb}}$ values and modified by the polynomial fit to the UV scaling. We also show the bolometric light curve of PTF 12dam as inferred by Chen et al. (2015) and updated by T.-W. Chen et al. (2017, Erratum, in preparation). The temperature, radius, and bolometric luminosity evolution of PTF 12dam are compared to two other SLSNe: iPTF 13ajg (filled purple diamonds; Vreeswijk et al. 2014) and CSS 121015 (filled green squares; Benetti et al. 2014).

transmission curves) to obtain model magnitudes for each filter. These model magnitudes are fit to the observed magnitudes to obtain the best-fit $T_{\mathrm{bb}}$ and $R_{\mathrm{bb}}$. To allow for the absorption features in the HST spectrum to vary with time, we introduce a fit parameter that simply scales the strength of these absorption features. In particular, the normalized, scaled HST spectrum is calculated as flux $_{\mathrm{HST} \text {, norm,scaled }}=1-\left(1-\right.$ flux $\left._{\mathrm{HST}, \text { norm }}\right) \times$ scale factor. For example, in Figure 2, the solid lines show the best-fitting modified blackbody spectrum. In the left panel, at a phase within a day of the epoch when the HST spectrum was taken, the UV absorption scaling was found to be around unity, as expected. On the right, the best-fit scale factor is much stronger, almost a factor of three. The third panel of Figure 3 shows the best-fitting UV absorption scale factor for all epochs for which UV photometry is available; it is clearly increasing in time.

Interestingly, an increase in the UV absorption lines is also observed for iPTF 13ajg (Vreeswijk et al. 2014), one of the few
SLSNe I with good temporal coverage of the near-UV region where this can be measured. The increase is clear from Figure 4 of Vreeswijk et al. (2014), where the spectra with UV coverage have been normalized by the blackbody fits. Measuring the combined equivalent width of the iPTF 13ajg model spectra in the rest-wavelength range 2000-2800 $\AA$ for epochs 2-5, we obtain a linear increase in rest-frame equivalent width of $35 \%$ between phases -8 and +10 days. The corresponding increase in equivalent width (or increase in UV scale factor) for PTF 12dam over the same phase is very similar, 39\%. However, the rest-frame equivalent width over the range 2000-2800 $\AA$ at phase -8 days is about a factor of three smaller for PTF 12 dam than for iPTF 13ajg: $200 \AA$ for PTF 12dam versus $600 \AA$ for iPTF 13ajg. An increase in the near-UV absorption is not unexpected: as the ejecta cool down from initially being very hot, the fraction of ions in the lower ionization states increases, and these are thought to be responsible for the near-UV absorption lines (Mg II, C II, C III, Fe III). 
The blackbody fits to the PTF 12 dam photometry are consistently below the $J H K$ flux measurements. The absorption in the UV could potentially be related to this excess emission in the NIR, because the absorbed UV emission is expected to be reradiated at longer wavelengths. This effect is observed in Type Ia SNe: the flux absorbed at blue wavelengths due to Fe II and Co II is redistributed to the red, eventually leading to a NIR secondary maximum (e.g., see Figures 4 and 5 of Pinto \& Eastman 2000; Kasen \& Woosley 2007).

We investigate whether the NIR excess is related to the UV absorption in PTF 12dam by determining the absorbed UV flux over the rest-frame range 2000-3000 A, and the excess NIR emission over the rest-frame range $1-2.5 \mu \mathrm{m}$. For the determination of NIR excess, we approximate the observed spectrum with a low-order polynomial shown as dotted lines in Figure 2, and integrate over the flux difference between this spectrum and the best-fitting blackbody (solid line). The inferred ratio of near-UV absorption to NIR excess shows a smooth evolution in time, starting at a factor of two at a phase of -20 days and reaching a factor of four at around -5 days, after which it very slowly decreases to a factor between 3.5 and 4 at +15 days. The wavelength upper limit of integration for the NIR excess of $2.5 \mu \mathrm{m}$ is arbitrary; if we extend it to a longer wavelength the above factor of roughly four would decrease. We note that the NIR excess is apparent until a phase of +130 days, after which the blackbody fits are consistent with the optical through NIR flux measurements. The apparent additional NIR emission above the Planck fits could potentially be caused by photons being emitted at longer wavelengths following absorption in the UV part of the spectrum, but it is difficult to confirm this. The lack of NIR excess beyond +130 days can be explained by the $\mathrm{SN}$ temperature having decreased to around $5000 \mathrm{~K}$ at this point, resulting in very few available UV photons to be re-emitted in the NIR.

The evolutions of the resulting blackbody temperature, radius, UV absorption scale factor, and bolometric luminosity are listed in Table 2 and shown in Figure 3. The estimate of the bolometric luminosity includes a correction for the UV absorption as described by the straight-line fit to the scale factor shown in the third panel from the top. Adopting the temperature evolution estimated by the polynomial fit at early times, we also infer the blackbody radius from each $r$-band measurement before maximum light, which provides a rough estimate of the radius evolution at early times.

Integrating the bolometric light curve that we derive over the phase interval from -70 to +334 days results in an estimated total radiated energy of $E_{\text {rad }}=1.8 \times 10^{51} \mathrm{erg}$ for PTF 12dam. This value is similar to that found for other SLSNe, such as the Type I SNLS 06D4eu (Howell et al. 2013) and iPTF 13ajg (Vreeswijk et al. 2014) and the Type II CSS 121015 (Benetti et al. 2014).

\section{2. iPTF $13 d c c$}

Figure 4 shows the light curve of iPTF 13dcc. The $r$-band evolution displays an initial slow decline extending over at least 30 rest-frame days, after which the SLSN is rebrightening to reach its peak brightness around 60 rest-frame days after the initial detection. The main peak brightness of the polynomial fit through the $r$-band data (shown by the solid line in Figure 4) of $r=19.5 \mathrm{mag}$ is reached at a modified Julian date of $\mathrm{MJD}=56618.3$, corresponding to UTC 2013 November 22.3. Although there is a gap in the data around the time of
Table 2

Blackbody Temperature, Radius, and Bolometric Luminosity of PTF 12dam

\begin{tabular}{|c|c|c|c|}
\hline $\begin{array}{l}\text { Phase } \\
\text { (days) }\end{array}$ & $\left(10^{3} \mathrm{~K}\right)$ & $\begin{array}{c}R_{\mathrm{bb}} \\
\left(10^{15} \mathrm{~cm}\right)\end{array}$ & $\begin{array}{l}\log L_{\mathrm{bql}} \\
\left(\operatorname{erg~s}^{-1}\right)\end{array}$ \\
\hline-78.86 & $\ldots$ & $<0.19$ & $<42.49$ \\
\hline-75.24 & $\ldots$ & $<0.21$ & $<42.54$ \\
\hline-71.68 & $\ldots$ & $<0.21$ & $<42.51$ \\
\hline-68.86 & $\ldots$ & $<0.23$ & $<42.58$ \\
\hline-66.28 & $\ldots$ & $<0.42$ & $<43.07$ \\
\hline-25.71 & $14.69 \pm 0.52$ & $2.57 \pm 0.11$ & $44.21 \pm 0.07$ \\
\hline-23.76 & $14.46 \pm 0.47$ & $2.71 \pm 0.11$ & $44.24 \pm 0.06$ \\
\hline-19.69 & $13.13 \pm 0.64$ & $3.26 \pm 0.20$ & $44.25 \pm 0.09$ \\
\hline-17.06 & $13.32 \pm 0.77$ & $3.28 \pm 0.24$ & $44.28 \pm 0.10$ \\
\hline-14.89 & $13.17 \pm 0.77$ & $3.39 \pm 0.25$ & $44.29 \pm 0.11$ \\
\hline-13.22 & $12.99 \pm 0.71$ & $3.49 \pm 0.24$ & $44.29 \pm 0.10$ \\
\hline-10.37 & $13.11 \pm 0.66$ & $3.50 \pm 0.23$ & $44.31 \pm 0.09$ \\
\hline-6.92 & $12.85 \pm 0.65$ & $3.64 \pm 0.24$ & $44.31 \pm 0.09$ \\
\hline-5.87 & $12.23 \pm 0.56$ & $3.91 \pm 0.24$ & $44.30 \pm 0.09$ \\
\hline-4.54 & $12.16 \pm 0.55$ & $3.94 \pm 0.24$ & $44.30 \pm 0.08$ \\
\hline 1.44 & $11.71 \pm 0.51$ & $4.14 \pm 0.24$ & $44.28 \pm 0.08$ \\
\hline 5.89 & $11.39 \pm 0.45$ & $4.24 \pm 0.24$ & $44.25 \pm 0.08$ \\
\hline 8.45 & $11.07 \pm 0.43$ & $4.35 \pm 0.23$ & $44.23 \pm 0.08$ \\
\hline 9.69 & $11.04 \pm 0.43$ & $4.35 \pm 0.24$ & $44.23 \pm 0.08$ \\
\hline 10.39 & $10.99 \pm 0.43$ & $4.37 \pm 0.24$ & $44.22 \pm 0.08$ \\
\hline 14.42 & $10.59 \pm 0.40$ & $4.52 \pm 0.24$ & $44.19 \pm 0.07$ \\
\hline 19.26 & $10.27 \pm 0.45$ & $4.60 \pm 0.30$ & $44.16 \pm 0.09$ \\
\hline 29.34 & $9.53 \pm 0.41$ & $4.78 \pm 0.31$ & $44.07 \pm 0.08$ \\
\hline 40.21 & $8.83 \pm 0.42$ & $4.97 \pm 0.40$ & $43.98 \pm 0.10$ \\
\hline 58.20 & $7.68 \pm 0.33$ & $5.58 \pm 0.40$ & $43.86 \pm 0.09$ \\
\hline 72.52 & $7.18 \pm 0.35$ & $5.79 \pm 0.53$ & $43.78 \pm 0.10$ \\
\hline 79.85 & $7.12 \pm 0.30$ & $5.50 \pm 0.41$ & $43.72 \pm 0.09$ \\
\hline 105.98 & $6.79 \pm 0.29$ & $4.95 \pm 0.37$ & $43.55 \pm 0.09$ \\
\hline 134.35 & $5.81 \pm 0.20$ & $5.51 \pm 0.34$ & $43.38 \pm 0.07$ \\
\hline 140.65 & $5.98 \pm 0.26$ & $4.98 \pm 0.44$ & $43.34 \pm 0.10$ \\
\hline 155.05 & $6.27 \pm 0.32$ & $4.01 \pm 0.45$ & $43.24 \pm 0.11$ \\
\hline 168.56 & $6.46 \pm 0.37$ & $3.39 \pm 0.43$ & $43.14 \pm 0.13$ \\
\hline 181.16 & $6.27 \pm 0.32$ & $3.28 \pm 0.36$ & $43.06 \pm 0.11$ \\
\hline 222.56 & $5.67 \pm 0.28$ & $3.00 \pm 0.31$ & $42.81 \pm 0.11$ \\
\hline 224.44 & $5.66 \pm 0.28$ & $2.96 \pm 0.31$ & $42.80 \pm 0.11$ \\
\hline 231.57 & $5.81 \pm 0.31$ & $2.68 \pm 0.32$ & $42.76 \pm 0.12$ \\
\hline 239.73 & $5.75 \pm 0.30$ & $2.58 \pm 0.30$ & $42.71 \pm 0.12$ \\
\hline 246.99 & $5.50 \pm 0.24$ & $2.70 \pm 0.23$ & $42.67 \pm 0.09$ \\
\hline 250.37 & $5.75 \pm 0.19$ & $2.52 \pm 0.19$ & $42.69 \pm 0.08$ \\
\hline 257.69 & $5.81 \pm 0.29$ & $2.25 \pm 0.24$ & $42.61 \pm 0.11$ \\
\hline 281.11 & $5.48 \pm 0.41$ & $2.17 \pm 0.32$ & $42.47 \pm 0.15$ \\
\hline 334.29 & $4.30 \pm 0.35$ & $2.33 \pm 0.41$ & $42.12 \pm 0.17$ \\
\hline
\end{tabular}

the peak, we adopt this date and magnitude as the main peak time and $r$-band magnitude of iPTF $13 \mathrm{dcc}$. At this epoch, we determine a $K$-correction (see Hogg et al. 2002) from observed $r$ to rest-frame SDSS $g$ of $-0.4 \mathrm{mag}$, leading to an absolute $g$ band peak magnitude of $M_{g}$, peak $=-22.0$ (the distance modulus of iPTF $13 \mathrm{dcc}$ is $41.93 \mathrm{mag}$ ). The absolute $g$-band magnitude is plotted on the right-hand axis of Figure 4. At the redshift of iPTF $13 \mathrm{dcc}(z=0.431)$, the effective wavelengths of observed $r$ and rest-frame $g$ match very well, leading to a $K$ correction that is almost independent of the color evolution of the SN and with a magnitude depending mainly on the redshift: $K_{r g}=-2.5 \log (1+z)=-0.4$.

The apparent $r$-band brightness of the early excess emission is only a few tenths of a magnitude fainter than the brightness at peak, and the early decline suggests it probably was even brighter before. iPTF or other surveys did not observe this sky location during several months prior to our first detection, so we do not have any constraints on the early brightness 


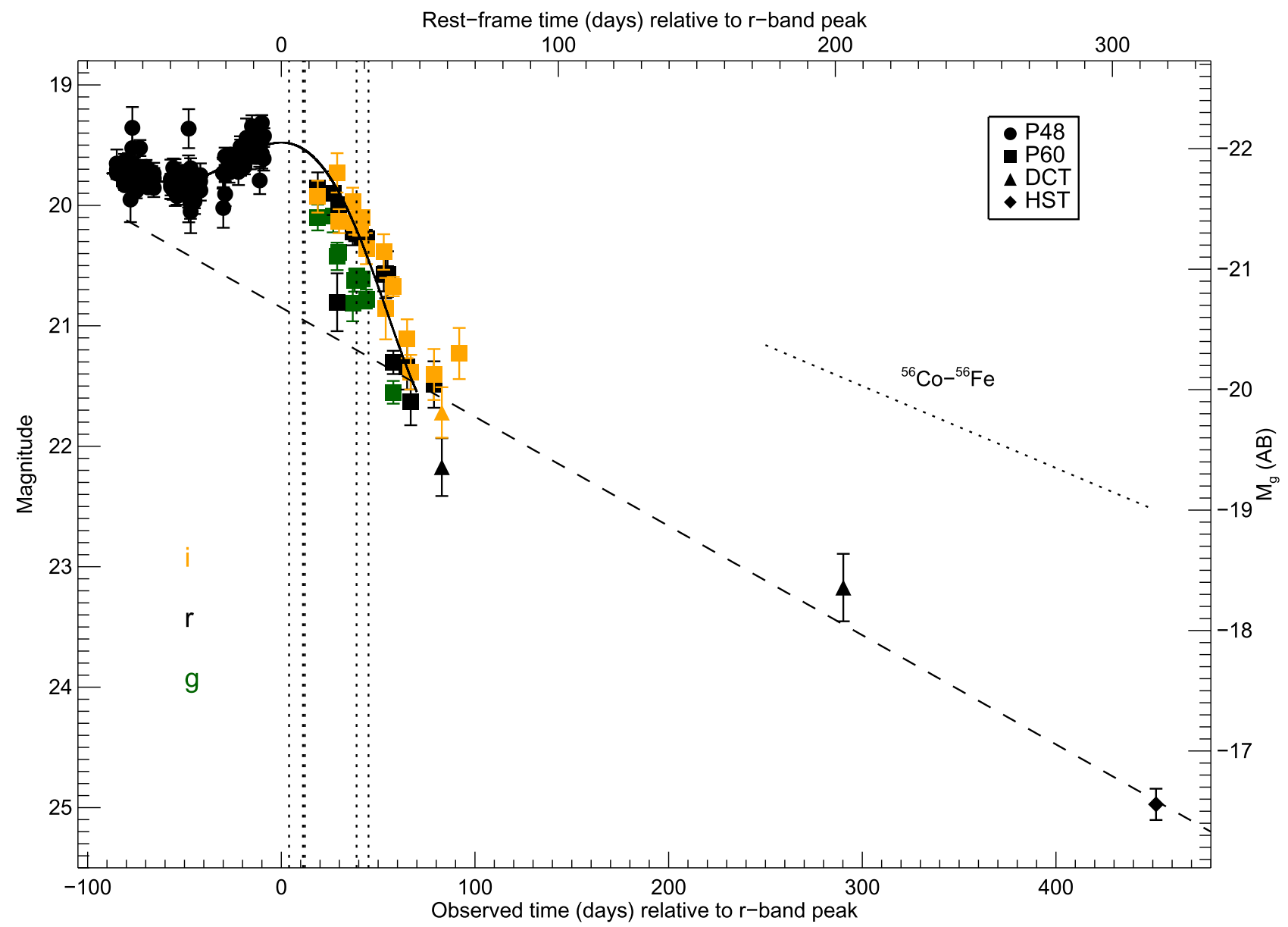

Figure 4. Light curves of iPTF 13dcc. Different colors and symbols denote different filters and telescopes, respectively. The solid line shows a polynomial fit to the $r$ band data up to about $t_{\mathrm{obs}}=70$ days. The dashed line displays a straight-line fit (i.e., exponential decay of the flux vs. linear time) to the last seven $r$-band data points. The late-time light curve is similar to the decay expected from radioactive decay of ${ }^{56} \mathrm{Co}$ to ${ }^{56} \mathrm{Fe}$, as indicated by the slanted dotted line. The vertical dotted lines show the epochs at which the iPTF $13 \mathrm{dcc}$ spectra were obtained.

evolution and the time of explosion. After the main peak, the light curve is declining rapidly until about 50 days after peak, followed by a very slow late-time decay with a slope that is only slightly steeper than that expected from the decay of radioactive ${ }^{56} \mathrm{Co}$, assuming full trapping of the gamma-ray photons produced.

Since we do not have any information on the evolution of the iPTF 13dcc light curve before our first detection, it is possible that the main peak occurred earlier and that the peak we consider to be the main peak is a late-time rebrightening. Such a rebrightening has been detected in other SLSNe, such as SN2015bn (Nicholl et al. 2016). However, in the case of iPTF 13dcc, the current main peak reaches an absolute $g$-band magnitude of $M_{g}=-22.0$. If this is a late-time rebrightening similar to that of SN2015bn, the main peak would have reached around $M_{g} \approx-23$, which would be stretching the brightness budget of SLSNe. Also, we show below that the estimated temperature of $\mathrm{PTF} 13 \mathrm{dcc}$ at the presumed peak is very similar to that of various other SLSNe I at peak. If indeed the actual main peak occurred before our first detection, we would have expected the temperature at the time of this supposed rebrightening to be well below $10,000 \mathrm{~K}$. For these reasons, we consider it unlikely that the actual main peak occurred before our first detection.

The light curve of iPTF 13dcc is rather limited in multifilter observations, making it difficult to derive a bolometric light curve from the photometry as we did for PTF 12dam. Instead, we turn to the spectroscopic sequence that was secured for this source as part of the iPTF follow-up campaign; see Table 1 and Figure 5. The times at which the five iPTF $13 \mathrm{dcc}$ spectra were taken, ranging from around the main peak until +30 days in the rest frame, are indicated by the vertical dotted lines in Figure 4. For each spectrum, we fit a Planck function to selected wavelength regions of the continuum free from obvious features (these regions are indicated with the short dashes at the top of the figure). This results in a best-fit blackbody temperature, radius, and corresponding bolometric luminosity at each epoch, shown by the filled black circles in the top, middle, and bottom panels of Figure 6, respectively. These values correspond to the bold-faced entries in Table 3. The iPTF $13 \mathrm{dcc}$ spectra and the blackbody fits are shown in Figure 5.

The temporal range spanned by the spectra is limited, from the time of the main peak to +30 rest-frame days. Therefore, to estimate the full bolometric light curve we have to make an assumption about the temperature evolution of iPTF $13 \mathrm{dcc}$ outside this range. In the top panel of Figure 6, we also show the inferred temperature evolution of PTF 12dam (derived in Section 3.1), CSS 121015 (Benetti et al. 2014), iPTF 13ajg (Vreeswijk et al. 2014), and DES 14X3taz (Smith et al. 2016). The temperature derived from the iPTF 13dcc spectra appears to match the evolution of PTF 12dam quite well. However, it is 


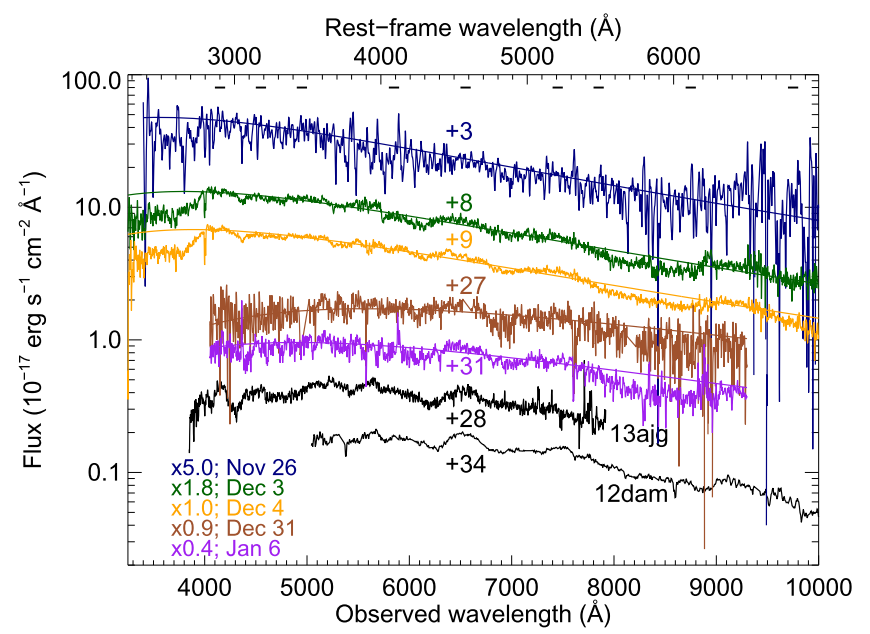

Figure 5. Time series of spectra of iPTF $13 \mathrm{dcc}$ (see Table 1). The spectra were corrected for Galactic extinction (Schlafly \& Finkbeiner 2011) and scaled to the polynomial fit to the $r$-band photometry depicted in Figure 4 to ensure a proper absolute calibration. To avoid the spectra overlapping each other, an additional scaling was applied as indicated at the bottom left, along with the date of observation (2013 November/December and 2014 January); the phase (restframe days relative to the $r$-band maximum) is shown next to each spectrum. The iPTF 13dcc spectra were smoothed with a Gaussian filter having an FWHM intensity of $5 \AA$. The spectra were fit with a Planck function to selected wavelength regions $50 \AA$ wide (the same for all spectra; these regions are indicated with dashes at the top of the plot). For comparison, we show the spectra of two known SLSNe I at the bottom in black: iPTF 13ajg (Vreeswijk et al. 2014) and PTF 12 dam (Nicholl et al. 2013) at phases of +28 and +34 days, respectively. The data used to create this figure are available.

also not too far from the temperature evolution of the other SLSNe I that are shown.

Assuming a particular temperature evolution and that iPTF 13 dcc is radiating as a blackbody, our $r$-band photometry can be used to infer the radius evolution. This is done assuming the temperature evolution of different SLSNe: PTF 12dam, iPTF 13ajg, and DES 14X3taz. The resulting radius evolution for each of these three cases is shown by the solid lines in the middle panel of Figure 6 . The radius evolution adopting the temperature of DES 14X3taz appears contrived; if the temperature evolved that dramatically for iPTF $13 \mathrm{dcc}$ as well, we would have detected it in our $r$-band photometry. In principle, it is possible that the temperature evolution of iPTF $13 \mathrm{dcc}$ contained a similar dramatic drop and rise to DES 14X3taz, but if it did that must have occurred before our first $r$-band detection. The middle panel also shows the radii inferred directly from the iPTF 13 dcc spectra (filled black circles), the radius evolution inferred for PTF 12dam (dashed black line, see Section 3.1), and that inferred for DES 14X3taz by Smith et al. (2016).

The bottom panel of Figure 6 shows the bolometric luminosity evolution of iPTF $13 \mathrm{dcc}$, adopting the temperature evolution of PTF 12dam, iPTF 13ajg, and DES 14X3taz. As mentioned above, the latter appears contrived and we consider it irrelevant for iPTF $13 \mathrm{dcc}$, whereas adopting the temperature evolution of iPTF 13ajg leads to unreasonably large values for the early-time bolometric luminosity of iPTF 13dcc. We therefore adopt the temperature evolution of PTF 12dam to derive the bolometric light curve of iPTF $13 \mathrm{dcc}$, listed in Table 3 and shown by the open circles in the bottom panel of Figure 6. We will adopt this bolometric evolution of iPTF 13dcc when performing the modeling in Section 4.
Integrating this bolometric light curve over the phase interval from -59 to +60 days $(-59$ to +315 days $)$ results in an estimated total radiated energy of $E_{\text {rad }}=2.8 \times 10^{51}$ erg $\left(3.0 \times 10^{51} \mathrm{erg}\right)$ for iPTF $13 \mathrm{dcc}$, which is about $60 \%$ larger than the value we derived for PTF 12dam (see Section 3.1). For comparison, we also show the bolometric evolution of PTF 12dam, CSS 121015, iPTF 13ajg, and DES 14X3taz.

Finally, we also plot the pair-instability supernova (PISN) model denoted R190 from Dessart et al. (2012), scaled up in luminosity $(\times 15)$ and shifted and contracted in time $(\times 0.45)$ to best match the bolometric behavior of iPTF 13dcc. The time of the main peak (i.e., at phase $=0$ in the figure) of the unscaled R190 model is about 220 days after explosion, or 100 days in the contracted model shown. Dessart et al. (2012) focus on three types of PISN, with the stars exploding being a red supergiant, a blue supergiant, or a Wolf-Rayet star (see also Dessart et al. 2013). The R190 model refers to a $190 M_{\odot}$ mainsequence star dying as a $164 M_{\odot}$ red supergiant with a surface radius of about $4000 R_{\odot}$ and an extended hydrogen envelope. Despite the R190 model being scaled in both luminosity and time, the similarity in the general brightness evolution to iPTF 13dcc is intriguing. However, this model with a hydrogen envelope is not the most natural for explaining the observations of a hydrogen-poor SLSN. In addition, Jerkstrand et al. (2016) compare their PISN models with the nebular spectra of SN 2007bi and PTF 12dam, finding discrepancies for several key observables and thus no support for a PISN interpretation.

\section{MODELING}

In this section, we explore different light-curve models that could explain the evolution of the bolometric light curves of PTF 12dam and iPTF 13dcc derived in the previous section. We start by applying the semianalytical light-curve models described by Chatzopoulos et al. (2012, 2013). These include the following three independent power inputs: (1) radioactive decay of ${ }^{56} \mathrm{Ni}$ and ${ }^{56} \mathrm{Co}$, (2) magnetar spin-down, and (3) forward- and reverse-shock heating due to SN ejecta interacting with CSM. The radioactive decay and interaction power inputs have also been combined by Chatzopoulos et al. (2012) into a hybrid model in which the two processes can be modeled simultaneously. These models are described in more detail below, but we also refer the reader to the above-mentioned papers.

In addition, we investigate whether the early-time bump or plateau observed in PTF 12dam and iPTF 13dcc could be produced by cooling emission from material heated by the shock produced in the explosion. For standard $\mathrm{SNe}$, the expected cooling emission has been modeled with the material being the outer part of the stellar envelope (Rabinak \& Waxman 2011) and also for the case in which an extended low-mass envelope surrounds a compact core (Nakar \& Piro 2014).

Nakar \& Piro (2014) show that a standard progenitor cannot explain the fast drop in bolometric luminosity after the first peak, which is supported by comparison of the model of Rabinak \& Waxman (2011) to observations of SLSNe with double-peaked light curves: LSQ 14bdq (Nicholl et al. 2015) and DES 14X3taz (Smith et al. 2016). Although Sapir \& Waxman (2016) extend the analytic expressions of Rabinak \& Waxman to later times and do infer a drop in luminosity for normal SNe II, their model is strictly valid only at times when 


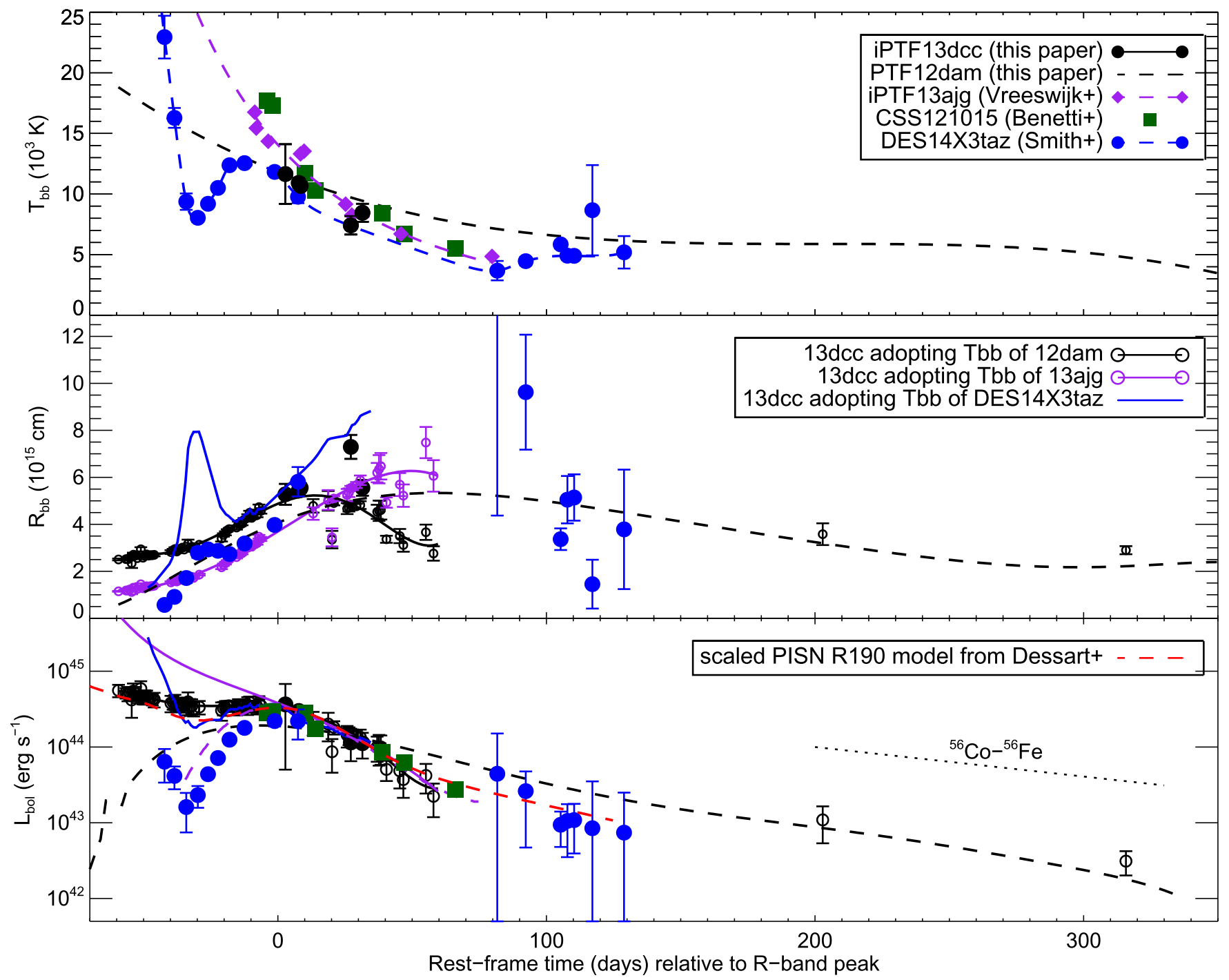

Figure 6. Time evolution of (from top to bottom) the blackbody temperature, radius, and bolometric luminosity of iPTF 13dcc. The filled black circles in all panels correspond to the best-fit values inferred from the Planck-function fits to the spectra, with fit parameters $T_{\mathrm{bb}}$ and $R_{\mathrm{bb}}$. Top panel: the temperature evolution of iPTF 13dcc is compared to that inferred for PTF 12dam (open black triangles and dashed line), CSS 121015 (green squares; Benetti et al. 2014), iPTF 13ajg (purple diamonds; Vreeswijk et al. 2014), and DES 14X3taz (filled blue circles; Smith et al. 2016). Middle panel: the radius evolution of iPTF 13dcc inferred from our $r$-band observations, adopting the temperature evolution of PTF 12dam (small open black circles and solid line), iPTF 13ajg (small open purple circles and solid line), and DES 14X3taz (solid blue line). The filled blue circles show the radius evolution inferred for DES 14X3taz by Smith et al. (2016). The black dashed line shows the radius evolution that we inferred for PTF 12dam (see Figure 3). Bottom panel: the iPTF 13dcc bolometric light curve inferred from the polynomial fits to the radius and assuming the temperature evolution of PTF 12dam (open black circles), iPTF 13ajg (solid purple line), and DES 14X3taz (solid blue line). These are compared with the evolution inferred for PTF 12dam (dashed black line), CSS 121015 (filled green squares), iPTF 13ajg (dashed purple line), DES 14X3taz (filled blue circles), and the pair-instability supernova (PISN) R190 model from Dessart et al. (2012), scaled and shifted to match the peak of iPTF 13dcc (red dashed line).

the photospheric (i.e., effective) temperature is above the recombination temperature necessary for the opacity in the relevant outer shells of the envelope to be time- and spaceindependent. For hydrogen-poor envelopes this latter temperature is of the order of the recombination temperature of doubly-ionized helium: $2-2.5 \mathrm{eV}$ or $23-29 \mathrm{kK}$, and so this model is not applicable to PTF 12dam or iPTF 13dcc, where the peak in early excess emission occurs at around 10 days, by which time the effective temperature has already dropped well below this temperature limit. For both LSQ 14bdq and DES 14X3taz, shock cooling of an extended envelope appears to describe the initial peak rather well (Piro 2015; Smith et al. 2016). We therefore focus on the case of an extended envelope, following the prescription of Piro (2015). We note that this model also successfully reproduces the early bump of
iPTF 15dtg, a spectroscopically normal SN Ic from a massive progenitor (Taddia et al. 2016).

Finally, we combine the Piro model with the Chatzopoulos magnetar and CSM interaction models to fit the entire bolometric light curve of iPTF $13 \mathrm{dcc}$. In the CSM case, this has the advantage that two fit parameters are the same in these models: the total SN energy, and the sum of Piro's core and extended mass is the same as the ejecta mass in the CSM interaction model. For PTF 12dam the early excess emission has too few data points for us to attempt this combined modeling. As a comparison for the best-fit values inferred for PTF 12dam and iPTF 13dcc, we also fit all the abovementioned models, including the combined ones, to the bolometric light curve of DES 14 X3taz presented by Smith et al. (2016). 
Table 3

Blackbody Temperature (Adopted from PTF 12dam), Radius, and Bolometric Luminosity of iPTF $13 \mathrm{dcc}$

\begin{tabular}{|c|c|c|c|}
\hline $\begin{array}{l}\text { Phase } \\
\text { (days) }\end{array}$ & $\left(10^{3} \mathrm{~K}\right)$ & $\begin{array}{c}R_{\mathrm{bb}} \\
\left(10^{15} \mathrm{~cm}\right)\end{array}$ & $\underset{\left(\operatorname{erg~s} L^{-1}\right)}{\log }$ \\
\hline-59.33 & 18.80 & $2.50 \pm 0.04$ & $44.75 \pm 0.07$ \\
\hline-56.54 & 18.39 & $2.52 \pm 0.03$ & $44.71 \pm 0.06$ \\
\hline-55.82 & 18.29 & $2.51 \pm 0.05$ & $44.70 \pm 0.08$ \\
\hline-55.17 & 18.19 & $2.58 \pm 0.05$ & $44.71 \pm 0.08$ \\
\hline-54.43 & 18.08 & $2.34 \pm 0.20$ & $44.62 \pm 0.15$ \\
\hline-53.79 & 17.99 & $2.71 \pm 0.09$ & $44.74 \pm 0.10$ \\
\hline-53.03 & 17.89 & $2.67 \pm 0.05$ & $44.72 \pm 0.08$ \\
\hline-52.32 & 17.79 & $2.56 \pm 0.04$ & $44.67 \pm 0.07$ \\
\hline-51.01 & 17.60 & $2.94 \pm 0.09$ & $44.77 \pm 0.10$ \\
\hline-50.24 & 17.49 & $2.58 \pm 0.04$ & $44.65 \pm 0.07$ \\
\hline-49.54 & 17.40 & $2.66 \pm 0.06$ & $44.66 \pm 0.08$ \\
\hline-48.83 & 17.30 & $2.72 \pm 0.04$ & $44.67 \pm 0.07$ \\
\hline-48.13 & 17.20 & $2.67 \pm 0.03$ & $44.65 \pm 0.06$ \\
\hline-47.40 & 17.10 & $2.69 \pm 0.03$ & $44.64 \pm 0.06$ \\
\hline-46.71 & 17.01 & $2.68 \pm 0.04$ & $44.63 \pm 0.07$ \\
\hline-46.04 & 16.92 & $2.70 \pm 0.06$ & $44.63 \pm 0.09$ \\
\hline-39.80 & 16.10 & $2.82 \pm 0.08$ & $44.58 \pm 0.10$ \\
\hline-39.02 & 16.00 & $2.89 \pm 0.06$ & $44.59 \pm 0.08$ \\
\hline-38.32 & 15.92 & $2.95 \pm 0.07$ & $44.60 \pm 0.09$ \\
\hline-37.63 & 15.83 & $2.84 \pm 0.06$ & $44.56 \pm 0.08$ \\
\hline-36.94 & 15.74 & $2.91 \pm 0.05$ & $44.57 \pm 0.08$ \\
\hline-36.25 & 15.66 & $2.97 \pm 0.05$ & $44.58 \pm 0.07$ \\
\hline-35.56 & 15.57 & $3.01 \pm 0.04$ & $44.58 \pm 0.07$ \\
\hline-34.86 & 15.49 & $2.93 \pm 0.05$ & $44.55 \pm 0.08$ \\
\hline-34.15 & 15.40 & $3.05 \pm 0.05$ & $44.57 \pm 0.08$ \\
\hline-33.45 & 15.31 & $3.17 \pm 0.17$ & $44.60 \pm 0.12$ \\
\hline-32.75 & 15.23 & $2.99 \pm 0.09$ & $44.54 \pm 0.10$ \\
\hline-32.06 & 15.15 & $3.04 \pm 0.08$ & $44.54 \pm 0.09$ \\
\hline-31.37 & 15.06 & $2.95 \pm 0.06$ & $44.50 \pm 0.08$ \\
\hline-29.26 & 14.82 & $3.13 \pm 0.06$ & $44.53 \pm 0.08$ \\
\hline-20.96 & 13.88 & $3.39 \pm 0.12$ & $44.48 \pm 0.10$ \\
\hline-20.26 & 13.81 & $3.55 \pm 0.07$ & $44.51 \pm 0.08$ \\
\hline-19.53 & 13.73 & $3.62 \pm 0.09$ & $44.52 \pm 0.09$ \\
\hline-18.85 & 13.66 & $3.74 \pm 0.05$ & $44.54 \pm 0.07$ \\
\hline-18.13 & 13.58 & $3.80 \pm 0.04$ & $44.54 \pm 0.06$ \\
\hline-15.39 & 13.29 & $3.86 \pm 0.09$ & $44.52 \pm 0.08$ \\
\hline-14.68 & 13.22 & $4.03 \pm 0.09$ & $44.55 \pm 0.08$ \\
\hline-13.98 & 13.15 & $4.00 \pm 0.08$ & $44.53 \pm 0.08$ \\
\hline-13.30 & 13.08 & $4.03 \pm 0.07$ & $44.53 \pm 0.07$ \\
\hline-12.59 & 13.01 & $4.19 \pm 0.07$ & $44.55 \pm 0.08$ \\
\hline-11.24 & 12.88 & $4.28 \pm 0.15$ & $44.56 \pm 0.10$ \\
\hline-10.52 & 12.80 & $4.49 \pm 0.08$ & $44.59 \pm 0.08$ \\
\hline-9.74 & 12.73 & $4.28 \pm 0.05$ & $44.53 \pm 0.06$ \\
\hline-9.03 & 12.66 & $4.35 \pm 0.07$ & $44.54 \pm 0.07$ \\
\hline-7.71 & 12.53 & $4.39 \pm 0.09$ & $44.53 \pm 0.08$ \\
\hline-7.02 & 12.47 & $4.73 \pm 0.11$ & $44.59 \pm 0.08$ \\
\hline-6.30 & 12.40 & $4.57 \pm 0.12$ & $44.55 \pm 0.09$ \\
\hline 2.81 & $11.65 \pm 2.46$ & $5.28 \pm 0.44$ & $44.56 \pm 0.27$ \\
\hline 7.81 & $\mathbf{1 0 . 9 1} \pm \mathbf{0 . 4 0}$ & $5.44 \pm 0.20$ & $\mathbf{4 4 . 4 8} \pm \mathbf{0 . 0 7}$ \\
\hline 8.50 & $10.66 \pm 0.34$ & $5.55 \pm 0.19$ & $44.45 \pm 0.06$ \\
\hline 13.16 & 10.73 & $4.79 \pm 0.29$ & $44.33 \pm 0.13$ \\
\hline 18.75 & 10.31 & $5.00 \pm 0.42$ & $44.30 \pm 0.15$ \\
\hline 20.16 & 10.20 & $3.35 \pm 0.37$ & $43.94 \pm 0.17$ \\
\hline 20.84 & 10.15 & $4.90 \pm 0.10$ & $44.26 \pm 0.08$ \\
\hline 25.80 & 9.81 & $4.67 \pm 0.23$ & $44.16 \pm 0.12$ \\
\hline 26.47 & 9.77 & $4.71 \pm 0.16$ & $44.16 \pm 0.10$ \\
\hline 27.15 & 9.72 & $4.83 \pm 0.07$ & $44.17 \pm 0.07$ \\
\hline 27.17 & $7.42 \pm 0.76$ & $7.29 \pm 0.51$ & $44.06 \pm 0.16$ \\
\hline 27.84 & 9.67 & $4.78 \pm 0.09$ & $44.15 \pm 0.08$ \\
\hline 29.26 & 9.58 & $4.77 \pm 0.11$ & $44.13 \pm 0.08$ \\
\hline 30.70 & 9.49 & $4.82 \pm 0.17$ & $44.13 \pm 0.10$ \\
\hline 31.48 & $8.45 \pm 0.74$ & $5.56 \pm 0.34$ & $44.05 \pm 0.14$ \\
\hline 36.98 & 9.10 & $4.53 \pm 0.30$ & $44.00 \pm 0.13$ \\
\hline
\end{tabular}

Table 3

(Continued)

\begin{tabular}{lccc}
\hline \hline $\begin{array}{l}\text { Phase } \\
\text { (days) }\end{array}$ & $\begin{array}{c}T_{\mathrm{bb}} \\
\left(10^{3} \mathrm{~K}\right)\end{array}$ & $\begin{array}{c}R_{\mathrm{bb}} \\
\left(10^{15} \mathrm{~cm}\right)\end{array}$ & $\begin{array}{c}\log L_{\text {bql }} \\
\left(\mathrm{erg} \mathrm{s}^{-1}\right)\end{array}$ \\
\hline 37.68 & 9.06 & $4.58 \pm 0.43$ & $44.00 \pm 0.16$ \\
38.37 & 9.02 & $4.60 \pm 0.40$ & $44.00 \pm 0.15$ \\
40.43 & 8.90 & $3.36 \pm 0.15$ & $43.70 \pm 0.11$ \\
45.41 & 8.63 & $3.50 \pm 0.30$ & $43.69 \pm 0.15$ \\
46.72 & 8.56 & $3.12 \pm 0.28$ & $43.57 \pm 0.15$ \\
55.12 & 8.15 & $3.66 \pm 0.33$ & $43.62 \pm 0.15$ \\
57.95 & 8.02 & $2.76 \pm 0.31$ & $43.35 \pm 0.17$ \\
202.87 & 5.88 & $3.58 \pm 0.46$ & $43.04 \pm 0.18$ \\
315.76 & 4.78 & $2.90 \pm 0.17$ & $42.49 \pm 0.13$ \\
\hline
\end{tabular}

Note. The bold-faced values are inferred from the iPTF $13 \mathrm{dcc}$ spectra, while for the others the PTF 12dam temperature evolution was adopted with the radii inferred using the $r$-band magnitude.

\subsection{PTF 12dam}

Figure 7 features the bolometric light curve of PTF 12dam as derived in Section 3. Around peak, the bolometric luminosity that we find is up to $50 \%$ higher than the bolometric luminosity evolution of PTF 12dam derived by Nicholl et al. (2013) and Chen et al. (2015). In private communication with the first authors of these papers to try to understand this discrepancy, it was found that they used the wrong photometric zero-points to convert the Swift UV magnitudes to fluxes (the zero-point for Vega was used while the magnitudes published by Nicholl et al. and Chen et al. are in the $\mathrm{AB}$ system), leading to an underestimate of the true bolometric luminosity (see the Errata of T.-W. Chen et al. 2017, in preparation and Nicholl et al. $2013^{26}$ ). Correcting the bolometric light curve of Chen et al. for this calibration error brings it up to a similar luminosity level to our bolometric light curve. In Figure 7 we show the corrected bolometric light curve from the Erratum of T.-W. Chen et al. (2017 in preparation), which is still slightly below our light curve around peak and up to a phase of 100 days.

We fit the Chatzopoulos models to our light-curve data (the filled circles shown in Figure 7) using a custom IDL $\chi^{2}$ minimization program, in which we make grateful use of the MPFIT procedures ${ }^{27}$ written by Craig Markwardt (Moré 1978; Markwardt 2009). Where relevant, we adopt a Thomson electron scattering opacity of $\kappa=0.2 \mathrm{~cm}^{2} \mathrm{~g}^{-1}$, appropriate for material without hydrogen, and also fit for the time of explosion, which is assumed to be within the phase range -70 to -65 days as constrained by the observations. We loosely limit the progenitor radius, $R_{0}$, to be within the range $0.1-500 R_{\odot}$, and use a fiducial value for the expansion velocity of $v_{\exp }=10,000 \mathrm{~km} \mathrm{~s}^{-1}$ for the radioactive decay and magnetar models (for the case of CSM interaction this velocity is not assumed but is calculated in the model).

The power input in the first model that we fit is radioactive decay of ${ }^{56} \mathrm{Ni}$ and ${ }^{56} \mathrm{Co}$, which produces energetic gamma-rays that are thermalized in the expanding ejecta. The fit parameters are the time of explosion $\left(t_{\text {expl, rest }}\right)$, the nickel mass produced $\left(M_{\mathrm{Ni}}\right)$, the mean of the hydrodynamical and diffusion timescales or effective light-curve timescale $\left(t_{\mathrm{d}}\right)$, a factor determining the fraction of gamma-rays that escape as a function of time

\footnotetext{
${ }^{26}$ Published online at http://www.nature.com/nature/journal/v539/n7630/ full/nature19850.html.

27 See http://purl.com/net/mpfit.
} 


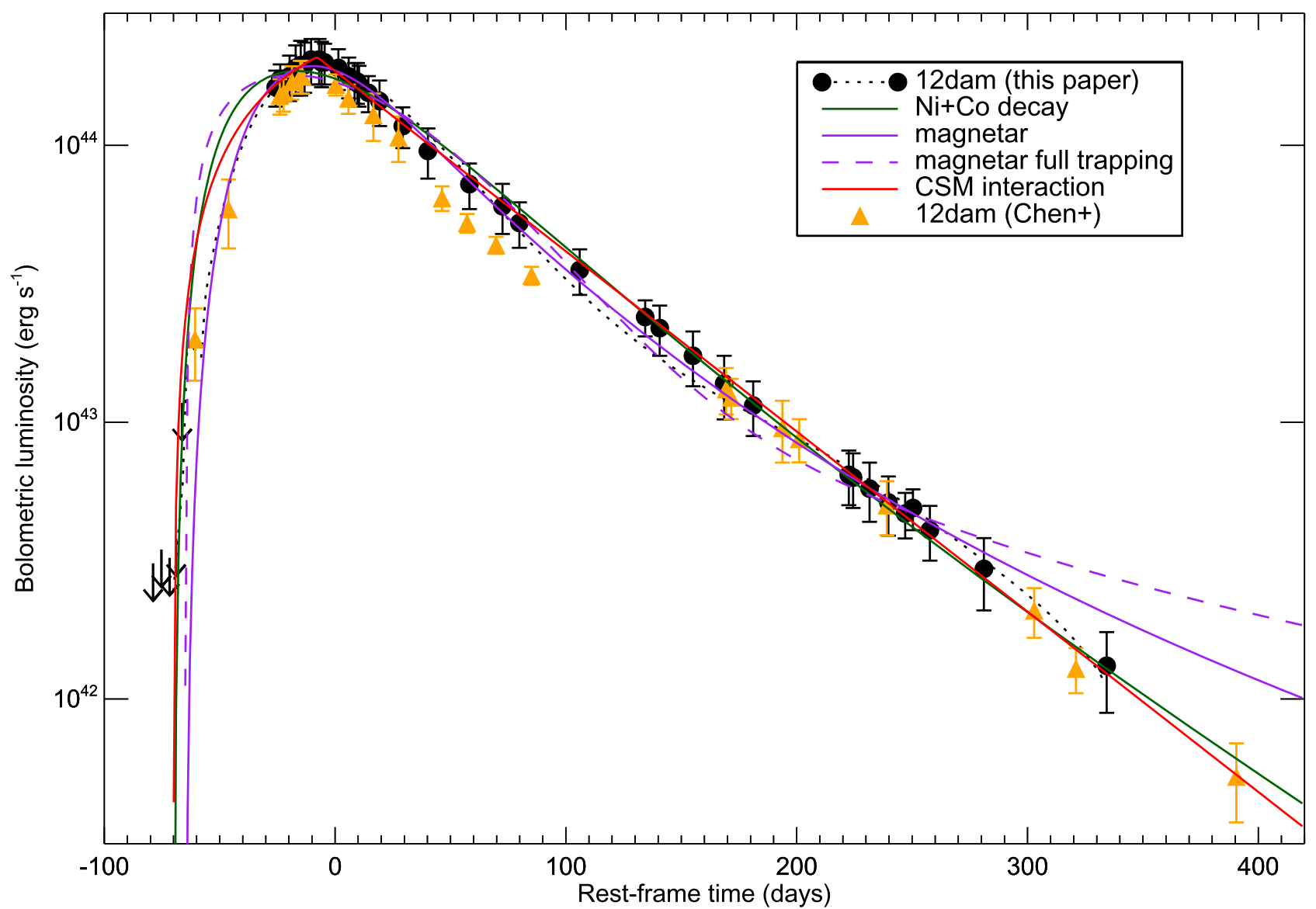

Figure 7. Model fits of radioactive decay (solid green line), magnetar (solid and dashed purple lines), and CSM interaction (solid red line) to the bolometric light curve of PTF 12dam that we derived in Section 3 (filled black circles and dotted line). See Tables 4-6 for the corresponding parameter best-fit values. All three models provide a good description of the PTF 12dam data. The magnetar fits including increased leakage of energy as a function of time and assuming full trapping are shown with the solid and dashed purple lines, respectively. The filled orange triangles show the bolometric light curve of PTF 12dam as inferred by Chen et al. (2015) and updated by T.-W. Chen et al. (2017, Erratum, in preparation).

Table 4

$\mathrm{Ni}+\mathrm{Co}$ Decay Best-fit Parameters

\begin{tabular}{lccc}
\hline \hline & PTF 12dam & iPTF 13dcc & DES 14X3taz \\
\hline$t_{\text {expl,rest }}($ days $)$ & $-70^{\mathrm{b}}$ & -89 & $-43^{\mathrm{b}}$ \\
$M_{\mathrm{Ni}}\left(M_{\odot}\right)$ & $21.9 \pm 0.5$ & $115 \pm 209$ & $26 \pm 39$ \\
$t_{\mathrm{d}}($ days $)$ & $46 \pm 2$ & $64 \pm 25$ & $61 \pm 48$ \\
$A_{\text {leakage }}\left(10^{3}\right.$ days $\left.^{2}\right)$ & $28 \pm 1$ & $3 \pm 8$ & $4 \pm 7$ \\
$R_{0}\left(R_{\odot}\right)$ & $0.1^{\mathrm{b}}$ & $0.1^{\mathrm{b}}$ & $0.1^{\mathrm{b}}$ \\
$\chi^{2} / \mathrm{DOF}$ & $5.9 / 32$ & $119 / 51$ & $73 / 10$ \\
\hline
\end{tabular}

Notes.

${ }^{a}$ For DES 14X3taz the first two detections have been discarded in the fit, focusing on fitting the main peak.

${ }^{b}$ Parameter reached the limit of the allowed range.

( $A_{\text {leakage }}$, modifying the luminosity by the factor $1-e^{-A t^{-2}}$; i.e., a large $A$ corresponds to full trapping), and the progenitor radius $\left(R_{0}\right)$. The goodness-of-fit $\chi^{2}$ value divided by the number of degrees of freedom (DOF) and the best-fit values for the $\mathrm{Ni}+\mathrm{Co}$ decay-model parameters are listed in Table 4 , and the corresponding best fit to the luminosity evolution of PTF 12 dam is shown by the solid green line in Figure 7.

This model describes the data very well. The very low reduced $\chi^{2}$ indicates that the uncertainties in the luminosity are likely overestimated. However, the best-fit nickel mass, $M_{\mathrm{Ni}}=22 M_{\odot}$, is much greater than the estimated ejecta mass,
$M_{\mathrm{ej}} \approx 8 M_{\odot}$, which is calculated using Equation (1) of Wheeler et al. (2015), repeated here:

$$
M_{\mathrm{ej}} \approx \frac{1}{2} \frac{\beta c}{\kappa} v_{\mathrm{exp}} t_{d}^{2}
$$

where we have substituted the rise time to maximum light $\left(t_{r}\right)$ with $t_{d}, \beta$ is a constant of integration (13.8), and $c$ is the speed of light. We note that this estimate of the ejecta mass is uncertain due to various assumptions made in its derivation, including the effective opacity and homologous expansion. Nonetheless, this model can probably be rejected on the basis that the nickel mass required to power the peak luminosity of PTF 12dam is unrealistically large (see also Nicholl et al. 2013; Chen et al. 2015). We note that Nicholl et al. (2013) inferred a lower nickel mass of 14-16 $M_{\odot}$ (see their extended data Figure 6) than we do, because they underestimated the true bolometric luminosity around peak of PTF 12dam, as we discussed above.

To check whether the light-curve tail can be powered by radioactive decay, we fit our late-time data beyond 100 restframe days. The nickel mass required $\left(12 M_{\odot}\right)$ is still large for the estimated ejecta mass $\left(22 M_{\odot}\right.$, which is larger than before because the best-fit effective diffusion timescale increased for this late-time fit). For the light curve beyond 200 days, the best nickel mass $\left(10 M_{\odot}\right)$ and estimated ejecta mass $\left(140 M_{\odot}\right)$ are compatible. Therefore, the radioactive decay of ${ }^{56} \mathrm{Ni}$ and ${ }^{56} \mathrm{Co}$ 
Table 5

Magnetar Best-fit Parameters

\begin{tabular}{|c|c|c|c|}
\hline & PTF 12dam & iPTF 13dcc & DES $14 \mathrm{X} 3 \mathrm{taz}^{\mathrm{a}}$ \\
\hline$t_{\text {expl,rest }}$ (days) & $-65^{b}$ & $-70^{b}$ & $-43^{b}$ \\
\hline$P_{\mathrm{sp}, \text { init }}(\mathrm{ms})$ & $2.30 \pm 0.03$ & $1.69 \pm 0.38$ & $1.8 \pm 2.8$ \\
\hline$B_{14}\left(10^{14} \mathrm{G}\right)$ & $0.7 \pm 0.7$ & $0.33 \pm 0.18$ & $0.39 \pm 0.68$ \\
\hline$t_{\mathrm{d}}$ (days) & $50 \pm 27$ & $10^{\mathrm{b}}$ & $49 \pm 202$ \\
\hline$A_{\text {leakage }}\left(10^{3}\right.$ days $\left.^{2}\right)$ & $32 \pm 51$ & $7 \pm 4$ & $3 \pm 11$ \\
\hline$R_{0}\left(R_{\odot}\right)$ & $0.1^{\mathrm{b}}$ & $0.1^{\mathrm{b}}$ & $0.1^{\mathrm{b}}$ \\
\hline$\chi^{2} / \mathrm{DOF}$ & $5.9 / 31$ & $127 / 50$ & $33.7 / 9$ \\
\hline
\end{tabular}

Notes.

${ }^{a}$ For DES 14X3taz the first two detections have been discarded in the fit, focusing on fitting the main peak.

${ }^{\mathrm{b}}$ Parameter reached the limit of the allowed range.

has difficulty accounting for the full bolometric light curve of PTF 12dam, but it could be that it is powering the late-time light curve.

In the magnetar model, the energy input is produced by the spin-down of a rapidly spinning, highly magnetic neutron star, the remnant of the explosion. Although it is not yet clear how, if at all, a large fraction of the rotational and/or magnetic energy can be converted to radiation, in this model it is assumed that the energy from the magnetar is thermalized in the expanding ejecta. The parameters of the magnetar model are the time of explosion ( $\left.t_{\text {expl, rest }}\right)$, the initial spin period of the neutron star in milliseconds $\left(P_{\mathrm{sp}, \text { init }}\right)$, its magnetic field strength in units of $10^{14}$ gauss $\left(B_{14}\right)$, the effective light-curve timescale $\left(t_{\mathrm{d}}\right)$, a constant $A_{\text {leakage, }}$, and the progenitor radius $\left(R_{0}\right)$. The parameters' best-fit values are listed in Table 5, and the corresponding best-fit model to the bolometric light curve of PTF 12dam is shown by the solid purple line in Figure 7.

Although it is not present in Equation (2) of Chatzopoulos et al. (2013), we include a leakage factor in exactly the same way as is done for the radioactive decay model (see also Wang et al. 2015). We stress that when assuming full trapping (i.e., a large value for $A_{\text {leakage }}$ ), the magnetar model predicts a late-time luminosity brighter than the observations, as already found by Chen et al. (2015). We show this full-trapping model fit with the dashed purple line in Figure 7, which has best-fit values $P_{\text {sp,init }}=1 \mathrm{~ms}$ (reaching the lower limit of the allowed range) and $B_{14}=1.63 \pm 0.03 \mathrm{G}\left(\chi^{2} / \mathrm{DOF}=\chi_{\nu}^{2}=0.7\right)$. The magnetar model including the leakage factor fits our data well $\left(\chi_{\nu}^{2}=0.2\right)$. In the radioactive decay model, the leakage is understood: the opacity of the gamma-rays produced in the radioactive decay of nickel and cobalt is decreasing with time, allowing an increasing fraction of the energy to escape. In the magnetar model, however, it is not clear how the rotational and/or magnetic energy is transferred to the expanding ejecta. It might also be in the form of high-energy photons, in which case the leakage would occur in a similar way to that in the radioactive decay model. We note that the initial spin period that we find $\left(P_{\mathrm{sp} \text {,init }}=2.30 \pm 0.03 \mathrm{~ms}\right)$ is shorter than that inferred by Chen et al. $\left(2015, P_{\mathrm{sp} \text {,init }}=2.72 \mathrm{~ms}\right)$, which moves the location of PTF 12dam in the plot of spin period versus host metallicity shown in Figure 7 of Chen et al. (2016) slightly away from the suggested relation.

The energy input in the CSM interaction model originates from the SN ejecta interacting with dense CSM (e.g., a dense progenitor wind or circumstellar shell previously cast off by the
Table 6

CSM Interaction Best-fit Parameters ${ }^{\mathrm{a}}$

\begin{tabular}{|c|c|c|c|}
\hline & PTF 12dam & iPTF 13dcc & $\overline{\text { DES } 14 \mathrm{X} 3 \operatorname{taz}^{\mathrm{b}}}$ \\
\hline$t_{\text {expl,rest }}$ (days) & $-70^{\mathrm{c}}$ & -79 & $-43^{c}$ \\
\hline$\delta$ & 0 & 2 & 2 \\
\hline$n$ & 8 & 6 & 14 \\
\hline$s$ & 0 & 0 & 0 \\
\hline$R_{\text {prog }}\left(R_{\odot}\right)$ & $0.1^{\mathrm{c}}$ & $0.1^{\mathrm{c}}$ & $0.1^{\mathrm{c}}$ \\
\hline$\rho_{\mathrm{CSM}}\left(10^{-13} \mathrm{~g} \mathrm{~cm}^{-3}\right)$ & 5 & 0.3 & 4 \\
\hline$M_{\mathrm{CSM}}\left(M_{\odot}\right)$ & 15 & 14 & 6 \\
\hline$M_{\text {ejecta }}\left(M_{\odot}\right)$ & 13 & 3 & 7 \\
\hline$E_{\mathrm{SN}}\left(10^{51} \mathrm{erg}\right)$ & 3 & 3 & 1 \\
\hline$\chi^{2} / \mathrm{DOF}$ & $1.8 / 31$ & $41.9 / 49$ & $16.4 / 8$ \\
\hline$R_{\mathrm{CSM}}^{\mathrm{d}}\left(10^{15} \mathrm{~cm}\right)$ & 2 & 6 & 2 \\
\hline$v_{\exp }{ }^{\mathrm{d}}\left(10^{3} \mathrm{~km} \mathrm{~s}^{-1}\right)$ & 15 & 25 & 15 \\
\hline
\end{tabular}

Notes.

a Owing to the complexity of the model, these best-fit values are very uncertain and should be considered as rough approximations.

${ }^{\mathrm{b}}$ For DES 14X3taz the first two detections have been discarded in the fit, focusing on fitting the main peak.

${ }^{c}$ Parameter reached the limit of the allowed range.

${ }^{\mathrm{d}}$ The lower two parameters are not fit, but are calculated within the model.

progenitor), resulting in a forward/circumstellar and a reverse/ ejecta shock. Chatzopoulos et al. (2013) use Chevalier (1982) and Chevalier \& Fransson (1994) to derive an expression for the time-dependent luminosity produced by the forward and reverse shocks, which are depositing kinetic energy into the CSM and SN ejecta, respectively. This is combined with the prescription of radiative diffusion developed by Arnett $(1980,1982)$ to derive an analytic expression for the output bolometric light curve (see Equation (20) of Chatzopoulos et al. 2013). The model has many free parameters: the time of the explosion $\left(t_{\text {expl,rest }}\right)$, the power-law index for the density profile of the inner and outer ejecta ( $\delta$ and $n$, respectively), the power-law index for the CSM density profile $(s)$, the progenitor radius $\left(R_{\text {prog }}\right)$, the density immediately outside the progenitor envelope $\left(\rho_{\mathrm{CSM}}\right)$, the CSM mass $\left(M_{\mathrm{CSM}}\right)$, the ejecta mass $\left(M_{\text {ejecta }}\right)$, and the total $\mathrm{SN}$ energy $\left(E_{\mathrm{SN}}\right)$. In our fitting routine, we loop over the following typical values for the parameters: $\delta=[0,2], n=6-14$, and $s=[0,2](s=0$ signifies a constant CSM density profile, such as that of a shell of material, while $s=2$ corresponds to that produced by a progenitor wind), and find the best-fit values for the remaining parameters. The bestfit values are listed in Table 6, and the corresponding best-fit model for PTF 12dam is shown by the solid red line in Figure 7.

The CSM interaction model provides an excellent fit to the data $\left(\chi_{\nu}^{2}=0.1\right)$, but this may not be so surprising when we consider the number of free parameters (see also Chatzopoulos et al. 2013). This CSM interaction model can be combined with the model for radioactive decay of nickel and cobalt, adding another free parameter, the nickel mass $\left(M_{\mathrm{Ni}}\right)$, but we fixed this to zero in the CSM fit.

We also fit the analytic shock-cooling model of an extended envelope surrounding the SN developed by Nakar \& Piro (2014) and Piro (2015) to the observed early-time $r$-band light curve of PTF $12 \mathrm{dam}$. The parameters are the time of the explosion $\left(t_{\text {expl }}\right)$, the core mass $\left(M_{\text {core }}\right)$, the energy in the $\mathrm{SN}$ shock (which is transferred to the extended material, $E_{\mathrm{SN}}$ ), and the mass, radius, and opacity of the extended material $\left(M_{\mathrm{ext}}\right.$, 
$R_{\text {ext }}$, and $\kappa$, respectively). The term $M_{\text {core }}$ is somewhat misleading; it refers to the part of the original stellar core that is ejected, i.e., the mass of the core minus the mass of the stellar remnant (see Nakar \& Piro 2014). We fix $\kappa=0.2 \mathrm{~cm}^{2} \mathrm{~g}^{-1}$ as for all the other models. We limit the SN energy and extended radius to be $E_{\mathrm{SN}} \leqslant 10^{53} \mathrm{erg}$ and $R_{\text {ext }} \leqslant 10^{14} \mathrm{~cm}$, respectively. The extended mass is constrained to be at most half the core mass, and the latter is required to be at least $1 M_{\odot}$. Given these parameters, the time evolution of the bolometric luminosity and radius of the extended material is provided by the model.

To fit the model to the observed magnitudes, we determine the effective temperature evolution based on the model parameters and transform the resulting rest-frame spectral blackbody flux to the observer's frame. We then extract observed model magnitudes by performing synthetic photometry on the spectrum using the $\mathrm{P} 48$-band transmission curve. To make use of the $r$-band upper limits nearest in time to the first detections, we converted the limits at observed times of about -73 and -76 days to expectation values of half the limit, with an uncertainty $(1 \sigma)$ the size of one sixth of the limit, so these added data points are consistent with the $3 \sigma$ upper limit. We did the same for the measurement at an observed time around -63 days, because by this time the luminosity is already dominated by whatever is powering the main peak. Although this is not clear in Figure 1, the $r$-band measurement at an observed time of -70 days is in fact composed of three different data points; hence, in the fit we use a total of seven measurements. Even when making use of the limits in the fit, the number of data points is limited. We therefore decided to fix the explosion energy to the best-fit value found for the CSM interaction fit. The resulting best-fit values are listed in Table 7. This fit is shown by the red solid line in the inset of Figure 1. As already noted, the number of DOF is very low (two), and so the parameters are not well constrained. However, this is just to show that the Piro model is capable of explaining the early-time evolution of the light curve of PTF 12dam with reasonable values for the parameters. For LSQ 14bdq, Piro (2015) finds acceptable fits with the following ranges of values for the fit parameters: $M_{\text {ext }}=0.3-0.5 M_{\odot}, R_{\text {ext }}=500-$ $5000 R_{\odot}\left(=3.5 \times 10^{13-14} \mathrm{~cm}\right)$, and $E_{\mathrm{SN}}=(9-55) \times 10^{51} \mathrm{erg}$.

\section{2. iPTF $13 d c c$}

Figure 8 shows the bolometric light curve of iPTF $13 \mathrm{dcc}$ as derived in Section 3. As we did for PTF 12dam, we fit the models of Chatzopoulos and Piro to our light-curve data. Again, we adopt a Thomson electron scattering opacity of $\kappa=0.2 \mathrm{~cm}^{2} \mathrm{~g}^{-1}$, and for iPTF $13 \mathrm{dcc}$ we assume that the time of explosion is in the phase range -100 to -70 days. We loosely limit the radius of the progenitor star, $R_{0}$, to be within the range $0.1-500 R_{\odot}$, and use a fiducial value for the expansion velocity of $10,000 \mathrm{~km} \mathrm{~s}^{-1}$ for the radioactive decay and magnetar models. The resulting best-fit values for the model parameters of the radioactive decay, magnetar, and CSM interaction models are listed in Tables 4, 5, and 6, respectively; the corresponding model fits are shown in the left panel of Figure 8. The amount of nickel required to power iPTF 13dcc is very large (albeit with an even larger formal uncertainty), much larger than the calculated mass in the ejecta $\left(M_{\mathrm{ej}} \approx 15 M_{\odot}\right)$, rendering this model unphysical. Also, the magnetar model is unable to describe both the early emission and main peak.
Table 7

Piro (2015) Best-Fit Parameters ${ }^{\mathrm{a}}$

\begin{tabular}{lccc}
\hline \hline & PTF 12dam & iPTF 13dcc & DES 14X3taz \\
\hline$t_{\text {expl, rest }}($ days $)$ & $-75^{\mathrm{b}}$ & $-70^{\mathrm{b}}$ & -45 \\
$M_{\text {core }}\left(M_{\odot}\right)$ & $1^{\mathrm{b}}$ & 35 & 4 \\
$E_{\mathrm{SN}}\left(10^{51} \mathrm{erg}\right)$ & $3^{\mathrm{c}}$ & $100^{\mathrm{b}}$ & $1^{\mathrm{c}}$ \\
$M_{\text {ext }}\left(M_{\odot}\right)$ & $0.5^{\mathrm{b}}$ & $17.5^{\mathrm{b}}$ & 0.4 \\
$R_{\text {ext }}\left(10^{13} \mathrm{~cm}\right)$ & 0.6 & $10^{\mathrm{b}}$ & $10^{\mathrm{c}}$ \\
$\chi^{2} / \mathrm{DOF}$ & $3.0 / 2$ & $52.7 / 17$ & $0.004 / 1$ \\
\hline
\end{tabular}

Notes.

${ }^{a}$ Owing to the degeneracy of the parameters in this model, these best-fit values are very uncertain and should be considered as rough approximations.

${ }^{\mathrm{b}}$ Parameter reached the limit of the allowed range.

${ }^{c}$ Parameter was held fixed; in the case of PTF 12dam and DES 14X3taz the explosion energy was fixed to the same value as the best-fit value of the CSM interaction model (see Table 6).

We also fit the analytic shock-cooling model of Piro (2015) to the bolometric light curve of iPTF 13dcc. As for PTF 12dam, we limit the $\mathrm{SN}$ energy and extended radius to be $E_{\mathrm{SN}} \leqslant 10^{53}$ erg and $R_{\text {ext }} \leqslant 10^{14} \mathrm{~cm}$, respectively, and the extended mass is constrained to be at most half the core mass. The best-fit values are given in Table 7 and the best fit is shown in the right panel of Figure 8. The line turns from solid to dotted at a phase of -25 days, beyond which the data points were excluded from this fit. The fit is good at early times, but undershoots the data points between phases of -45 and -25 days. A larger explosion energy of $1.5 \times 10^{53}$ erg would provide a very good fit, but this is unreasonably high. The inferred extended mass, which is the main parameter determining the width of the light curve, is much larger in the case of iPTF $13 \mathrm{dcc}\left(\sim 18 \mathrm{M}_{\odot}\right)$ than that needed to explain the excess emission in PTF 12 dam $\left(\sim 0.5 M_{\odot}\right)$.

Finally, we fit a combination of the Piro plus magnetar and also Piro plus CSM interaction models to the iPTF 13dcc observations. In the latter combined model, the explosion energy is set to the same value in both the Piro and CSM interaction models, and the ejecta mass (of the CSM interaction model) is set to the sum of the core mass and the extended mass (both of the Piro model): $M_{\text {core }}+M_{\text {ext }}=M_{\text {ejecta }}$. This is because the ejected core mass is sweeping up the extended mass, which is at a shorter distance than the CSM mass, and thus the sum of these masses equals the ejecta mass in the CSM interaction model. The best-fit parameters of these combination models are listed in Tables 8 and 9, and the corresponding light curves are shown in the right panel of Figure 8.

\subsection{DES $14 X 3$ taz}

Smith et al. (2016) present observations of the hydrogenpoor SLSN DES 14X3taz, which also shows evidence for early-time excess emission. These authors were able to catch this early emission in multiple filters, for the first time, allowing for a quite accurate determination of the temperature, radius, and bolometric light-curve evolution for the early-time emission. Since the bolometric evolution of this SLSN is so well constrained from a very early epoch, we also fit DES 14X3taz with the same models as we have done for PTF 12dam and iPTF 13dcc above. The filled black circles in Figure 9 show the bolometric light curve of DES 14X3taz as presented by Smith et al. (2016). The time of explosion for 

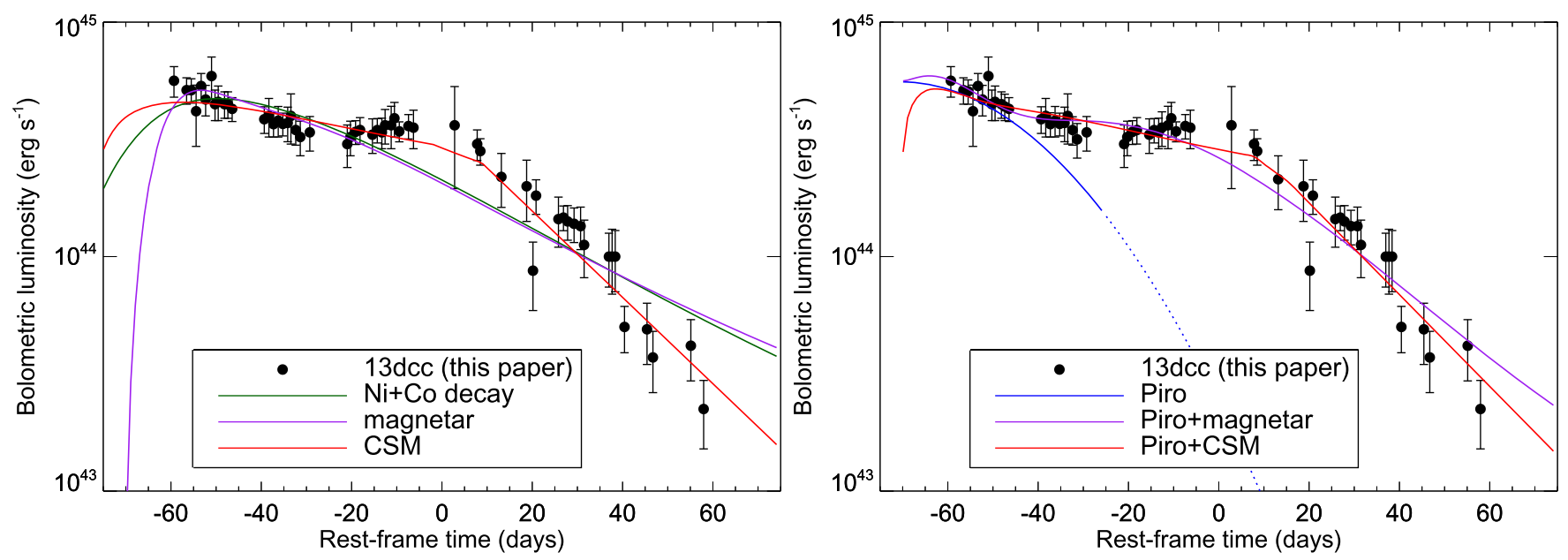

Figure 8. Left panel: model fits of radioactive decay, magnetar, and CSM interaction to the bolometric light curve of iPTF $13 \mathrm{dcc}$ (up to a phase of +80 days). While the radioactive decay and magnetar models fail to fit both the early excess emission and main peak, the CSM interaction model describes the observations rather well. Right panel: a Piro model fit to the early-time light curve (phase $<-25$ days), and a combined fit of the Piro plus magnetar and Piro plus CSM interaction models to the iPTF 13dcc light curve. The two breaks in the light curve of the CSM interaction model, seen in both panels, correspond to the times of termination of the forward and reverse shocks. See Tables 4-9 for the corresponding best-fit parameter values for all these model fits.

Table 8

Piro+magnetar Best-fit Parameters ${ }^{\mathrm{a}}$

\begin{tabular}{lcc}
\hline \hline & iPTF 13dcc & DES 14X3taz \\
\hline$t_{\text {expl,rest }}($ days $)$ & $-70^{\mathrm{b}}$ & $-43^{\mathrm{b}}$ \\
$M_{\text {core }}\left(M_{\odot}\right)$ & 12 & 1.2 \\
$M_{\text {ext }}\left(M_{\odot}\right)$ & 6 & $0.6^{\mathrm{b}}$ \\
$R_{\text {ext }}\left(10^{13} \mathrm{~cm}\right)$ & 4 & 0.2 \\
$E_{\mathrm{SN}}\left(10^{51} \mathrm{erg}\right)$ & $100^{\mathrm{b}}$ & 20 \\
$P_{\text {sp,init }}(\mathrm{ms})$ & $1.2 \pm 1.7$ & $1.8 \pm 2.8$ \\
$B_{14}\left(10^{14} \mathrm{G}\right)$ & $1.1 \pm 0.8$ & $0.4 \pm 0.7$ \\
$t_{\mathrm{d}}($ days $)$ & $66 \pm 72$ & $49 \pm 209$ \\
$A_{\text {leakage }}\left(10^{3}\right.$ days $\left.^{2}\right)$ & $11 \pm 37$ & $3 \pm 11$ \\
$R_{0}\left(R_{\odot}\right)$ & $0.1^{\mathrm{b}}$ & $0.1^{\mathrm{b}}$ \\
$\chi^{2} /$ DOF & $48.9 / 46$ & $34.2 / 7$ \\
\hline
\end{tabular}

Notes.

${ }^{a}$ Owing to the degeneracy of the Piro parameters in this model, these best-fit values for the Piro model are very uncertain and should be considered as rough approximations.

${ }^{\mathrm{b}}$ Parameter reached the limit of the allowed range.

DES 14X3taz is very well constrained by observations to be in the range -50 to -43 days. As for PTF 12 dam and iPTF 13dcc, we loosely limit the radius of the progenitor star, $R_{0}$, to be within the range $0.1-500 R_{\odot}$, and use a fiducial value for the expansion velocity of $10,000 \mathrm{~km} \mathrm{~s}^{-1}$. We exclude the first two detections and focus on fitting the models to the main peak, but constrain the time of explosion to be before the first detection. The resulting best-fit values for the model parameters of the radioactive decay, magnetar, and CSM interaction models are listed in Tables 4, 5, and 6, respectively. The corresponding evolution of the bolometric luminosity is shown in the left panel of Figure 9. As we did for iPTF 13dcc in the previous section, a combination of the Piro plus magnetar and Piro plus CSM interaction models is fit to the full light curve of DES 14X3taz. The best-fit parameters of these combination models are listed alongside those of iPTF 13dcc in Tables 8 and 9 , and the corresponding light curves of the bolometric model are shown in the right panel of Figure 9.
Table 9

Piro+CSM Interaction Best-fit Parameters ${ }^{\mathrm{a}}$

\begin{tabular}{lcc}
\hline \hline & iPTF 13dcc & DES 14X3taz \\
\hline$t_{\text {expl, rest }}($ days $)$ & $-70^{\mathrm{b}}$ & $-43^{\mathrm{b}}$ \\
$M_{\text {core }}\left(M_{\odot}\right)$ & 2.4 & 7.9 \\
$M_{\text {ext }}\left(M_{\odot}\right)$ & $1.2^{\mathrm{b}}$ & 0.1 \\
$R_{\text {ext }}\left(10^{13} \mathrm{~cm}\right)$ & $10^{\mathrm{b}}$ & 7 \\
$\delta$ & 2 & 0 \\
$n$ & 6 & 14 \\
$s$ & 0 & 0 \\
$R_{\text {prog }}\left(R_{\odot}\right)$ & 0.4 & $0.1^{\mathrm{b}}$ \\
$\rho_{\text {CSM }}{ }^{\mathrm{c}}\left(10^{-13} \mathrm{~g} \mathrm{~cm}^{-3}\right)$ & 0.3 & 5 \\
$M_{\mathrm{CSM}}\left(M_{\odot}\right)$ & 12 & 6 \\
$M_{\text {ejecta }}\left(M_{\odot}\right)$ & 3.6 & 8.0 \\
$E_{\mathrm{SN}}\left(10^{51} \mathrm{erg}\right)$ & 3 & 2 \\
$\chi^{2} / \mathrm{DOF}$ & $36.6 / 47$ & $16.6 / 8$ \\
$R_{\mathrm{CSM}}{ }^{\mathrm{c}}\left(10^{15} \mathrm{~cm}^{\mathrm{b}}\right)$ & 6 & 2 \\
$v_{\text {exp }}{ }^{\mathrm{c}}\left(10^{3} \mathrm{~km} \mathrm{~s}^{-1}\right)$ & 23 & 18 \\
\hline
\end{tabular}

Notes.

${ }^{\text {a }}$ Owing to the complexity of the model, these best-fit values are very uncertain and should be considered as rough approximations.

${ }^{\mathrm{b}}$ Parameter reached the limit of the allowed range.

${ }^{\mathrm{c}}$ The lower two parameters are not fit, but are calculated within the CSM interaction model.

\section{DISCUSSION}

Figure 10 shows a comparison of the absolute $g$-band light curves of iPTF 13dcc, LSQ 14bdq, SN 2006oz, DES 14X3taz, and PTF 12dam. The iPTF 13dcc data were derived by converting the observed $r$-band measurements to rest-frame $g$, where we computed the $K$-corrections adopting the temperature evolution of PTF 12dam (see Figure 6). Since the effective wavelengths of the observed $r$-band and rest-frame $g$-band filters match well at the redshift of iPTF 13dcc $(z=0.431)$, these $K$-corrections are very close to $-2.5 \log$ $(1+z)$ and do not depend very much on the assumed temperature evolution. For PTF 12dam $(z=0.107)$, we instead 

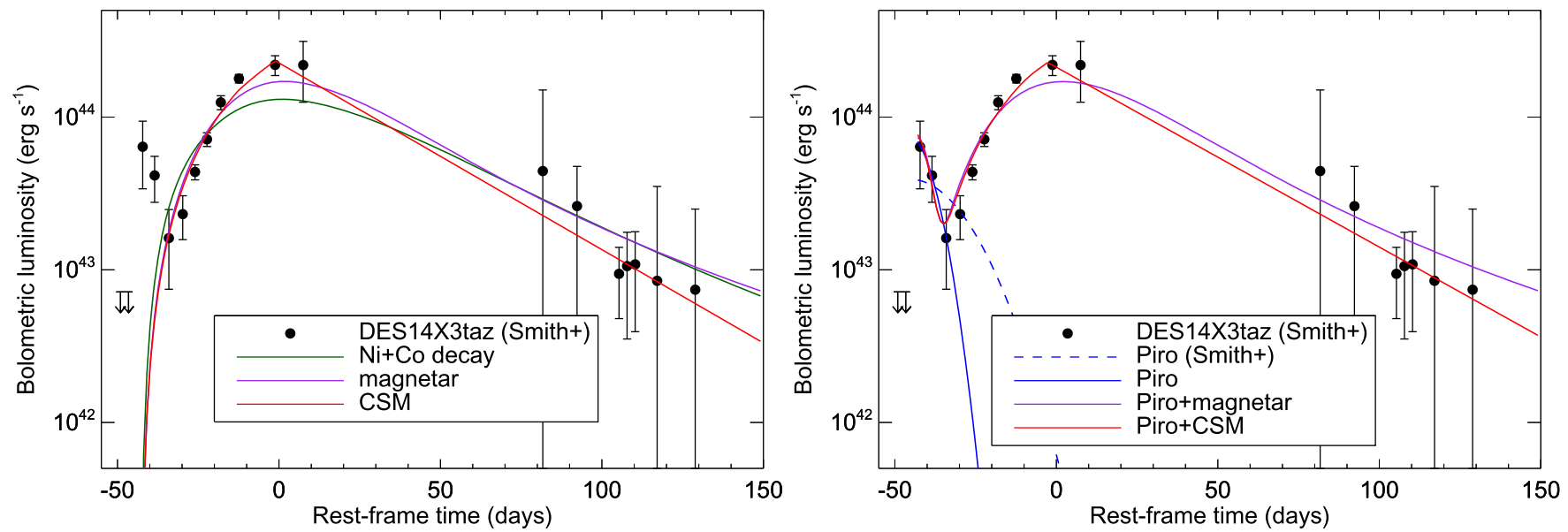

Figure 9. Model fits of radioactive decay, magnetar, CSM interaction, and the Piro model to the bolometric light curve of DES 14X3taz (Smith et al. 2016). In the left panel, we only fit the main peak, and the first two detections have been excluded from the fits. The radioactive decay model provides a poor fit, while the magnetar model fits the data reasonably well. The CSM interaction model provides the best match with the observations. On the right, we fit the Piro (2015) model to the first three detections, indicated with the solid blue line. For reference, we also show the model parameters found by Smith et al. (2016) from fitting the griz magnitudes rather than the bolometric luminosity as we do in this paper. We also perform combined Piro+magnetar and Piro+CSM interaction fits, shown by the solid lines in the right panel. See Tables 4-9 for the corresponding best-fit parameter values for all these model fits.

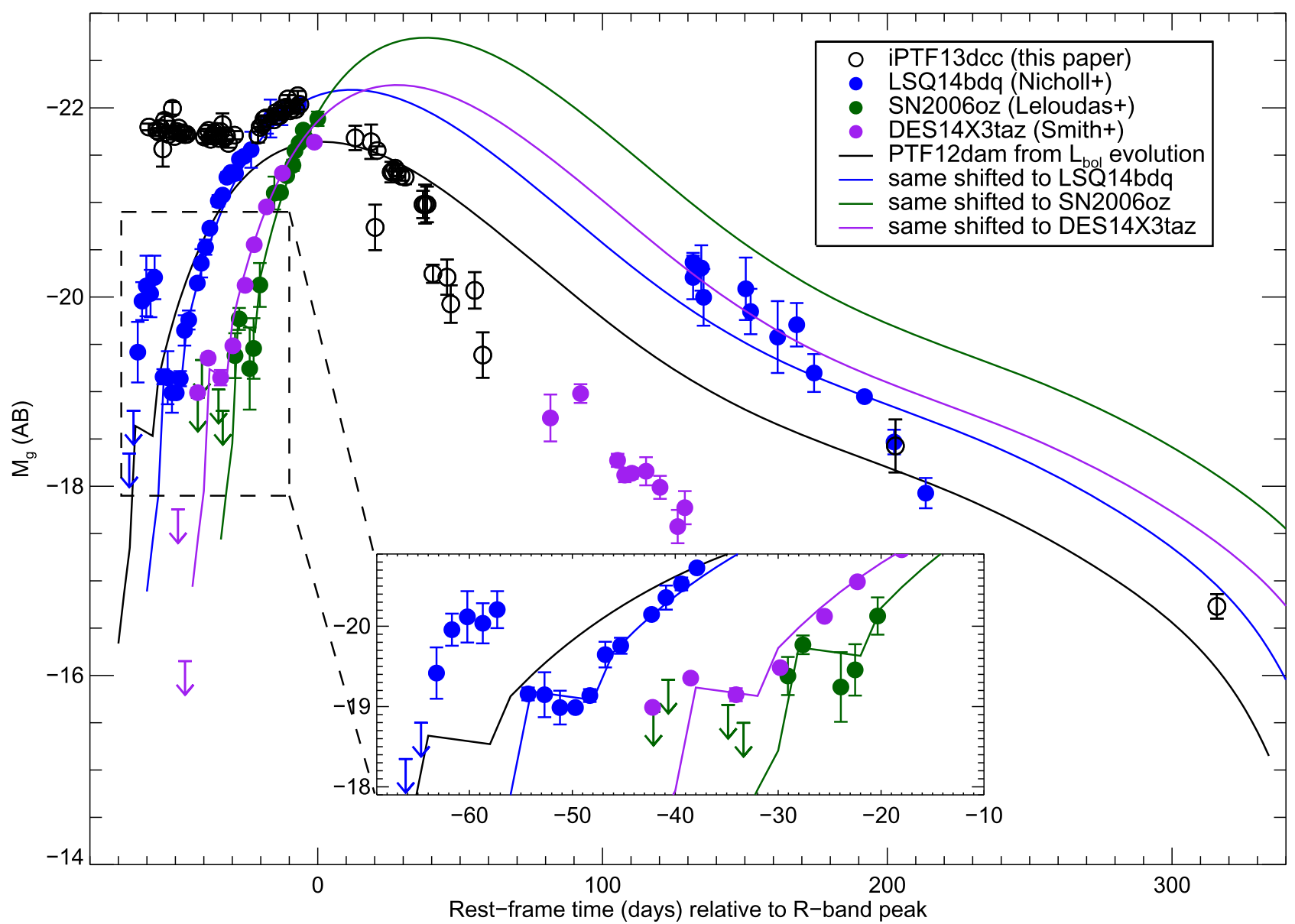

Figure 10. Absolute $g$-band light curves of iPTF 13dcc (open black circles), LSQ14bdq (filled blue circles; Nicholl et al. 2015), SN 2006oz (filled green circles; Leloudas et al. 2012), and DES 14X3taz (filled purple circles; Smith et al. 2016). The solid black line shows the evolution of PTF 12dam assuming the evolution of temperature and radius as inferred from the photometry and as shown in Figure 3. The blue, green, and purple lines correspond to the $M_{g}$ light curve of PTF $12 \mathrm{dam}$ simply shifted in time and magnitude to roughly match the data points of LSQ14bdq, SN 2006oz, and DES 14X3taz, respectively. The open black circles are the iPTF 13dcc $M_{g}$ values inferred from the observed P48 and P60 $r$-band magnitudes, applying a $K$-correction that is based on the temperature evolution of PTF 12dam (see Figure 6). 
Table 10

Log of Observations of PTF 12dam

\begin{tabular}{lcccc}
\hline $\begin{array}{l}\text { MJD } \\
\text { (days) }\end{array}$ & $\begin{array}{c}\text { Phase }^{\mathrm{a}} \\
\text { (days) }\end{array}$ & Telescope & Filter & $\begin{array}{c}\text { Magnitude }^{\mathrm{b}} \\
\text { AB }\end{array}$ \\
\hline 56009.34 & -78.92 & $\mathrm{P} 48$ & $R$ & $>22.26$ \\
56013.35 & -75.30 & $\mathrm{P} 48$ & $R$ & $>22.10$ \\
56017.29 & -71.73 & $\mathrm{P} 48$ & $R$ & $>22.13$ \\
56020.41 & -68.91 & $\mathrm{P} 48$ & $R$ & $>21.94$ \\
56023.28 & -66.33 & $\mathrm{P} 48$ & $R$ & $>20.69$ \\
56025.33 & -64.47 & $\mathrm{P} 48$ & $R$ & $20.11 \pm 0.17$ \\
56027.26 & -62.73 & $\mathrm{P} 48$ & $R$ & $20.02 \pm 0.12$ \\
56027.29 & -62.70 & $\mathrm{P} 48$ & $R$ & $20.11 \pm 0.11$ \\
56027.32 & -62.68 & $\mathrm{P} 48$ & $R$ & $20.04 \pm 0.10$ \\
56033.49 & -57.10 & $\mathrm{P} 48$ & $R$ & $19.89 \pm 0.08$ \\
\hline
\end{tabular}

Notes.

${ }^{\text {a }}$ Calculated using MJD $_{r \text {, peak }}=56096.7$ and $z=0.107$

$\mathrm{b}$ The magnitudes have not been corrected for Galactic extinction.

(This table is available in its entirety in machine-readable form.)

computed a continuous rest-frame $g$-band light curve by adopting the evolution of temperature and bolometric luminosity as derived in Figure 3. For each SLSN shown in Figure 10, except for iPTF 13dcc, we shifted the light curve of PTF 12dam, in both time and magnitude, to match the data points. This is simply to show how the observed early excess emission of each SLSN compares in duration and magnitude with that of the others. For both SN 2006oz and DES 14X3taz the match with PTF 12dam at early epochs is quite good, whereas at late times the DES 14X3taz light curve is dropping much more rapidly than that of PTF 12 dam. For LSQ 14bdq the early excess emission is longer and brighter than that of PTF 12dam, but at late times the two match surprisingly well. The obvious outlier is the light curve of iPTF 13dcc: its early emission is of a much longer duration and a few magnitudes brighter than those of the others.

Kasen et al. (2016) suggest that the early excess emission observed for LSQ 14bdq could be caused by a magnetar central engine whose energy input drives a shock through the preexploded SN ejecta, resulting in a burst of shock-breakout emission several days after the explosion. The radiation is expected to be released in the optical/UV wavelengths and to have a duration of several days, with the emission being dimmer than the main light-curve peak resulting from continued magnetar heating. As can be seen in Figure 7 of Kasen et al. (2016), for standard magnetar and ejecta parameters, the shock-breakout emission produces only a kink in the overall light curve. In principle, this model is capable of explaining the early excess emission in PTF 12 dam. However, the early emission that we observe for iPTF $13 \mathrm{dcc}$ is too bright and too extended to be accommodated in this model. Even when pushing the ejecta mass and kinetic energy to large values (see Figure 8 of Kasen et al. 2016), this model is not able to reproduce the iPTF 13 dcc observations.

Based on the model fits presented in Section 4, the CSM interaction model seems to provide better fits to the data. Moreover, the magnetar model is unable by itself to fit the precursor bumps seen in SLSNe but requires the addition of the Piro model (or similar) and the presence of extended material at large radii to explain the first peak. The combination of Piro and magnetar provides a reasonable description of the
Table 11

Log of Observations of iPTF $13 \mathrm{dcc}$

\begin{tabular}{lcccc}
\hline \hline $\begin{array}{l}\text { MJD } \\
\text { (days) }\end{array}$ & $\begin{array}{c}\text { Phase }^{\mathrm{a}} \\
\text { (days) }\end{array}$ & Telescope & Filter & $\begin{array}{c}\text { Magnitude }^{\mathrm{b}} \\
\mathrm{AB}\end{array}$ \\
\hline 56533.36 & -59.36 & $\mathrm{P} 48$ & $R$ & $19.68 \pm 0.12$ \\
56533.42 & -59.32 & $\mathrm{P} 48$ & $R$ & $19.73 \pm 0.06$ \\
56533.45 & -59.29 & $\mathrm{P} 48$ & $R$ & $19.76 \pm 0.05$ \\
56537.35 & -56.57 & $\mathrm{P} 48$ & $R$ & $19.66 \pm 0.06$ \\
56537.47 & -56.49 & $\mathrm{P} 48$ & $R$ & $19.86 \pm 0.04$ \\
56537.50 & -56.46 & $\mathrm{P} 48$ & $R$ & $19.70 \pm 0.04$ \\
56538.45 & -55.80 & $\mathrm{P} 48$ & $R$ & $19.80 \pm 0.04$ \\
56539.34 & -55.18 & $\mathrm{P} 48$ & $R$ & $19.66 \pm 0.07$ \\
56539.38 & -55.15 & $\mathrm{P} 48$ & $R$ & $19.79 \pm 0.07$ \\
56539.42 & -55.12 & $\mathrm{P} 48$ & $R$ & $19.80 \pm 0.06$ \\
\hline
\end{tabular}

Notes.

${ }^{\text {a }}$ Calculated using MJD $_{r, \text { peak }}=56618.3$ and $z=0.431$.

$\mathrm{b}$ The magnitudes have not been corrected for Galactic extinction.

(This table is available in its entirety in machine-readable form.)

iPTF 13dcc light curve, even though it does not fit the data well around peak and appears to overshoot the data beyond +40 days. Moreover, the explosion energy for this model reaches the upper end of the range that we allowed: $E_{\mathrm{SN}}=10^{53}$ erg. We find that the CSM interaction model, which in fact contains one free parameter less than the number used in the combined Piro and magnetar model, can provide a good fit to the light curve of iPTF 13dcc, even without the addition of the Piro model (see Table 6 and Figure 8). The first minimum in the light curve cannot be accurately reproduced (see the left panel of Figure 8), but a time-dependent opacity (we have assumed a constant opacity throughout this paper) could be invoked to explain the early light-curve shape. Also, this dip in the light curve of iPTF $13 \mathrm{dcc}$ is reminiscent of the picture proposed by Moriya \& Maeda (2012), where a drop in the light curve arises naturally. These authors suggest that such a dip is a solid prediction from the strong interaction scenario regardless of the power source for the early emission.

The ability of the CSM interaction model to reproduce the light curves of many SLSNe can be explained by the fact that it includes many free parameters. In addition, the CSM model by Chatzopoulos et al. (2012, 2013) includes a number of simplifying assumptions, of which the most important are those of a central power source and a stationary photosphere, allowing for the use of Arnett-style diffusion by using analytical equations. However, it is not clear whether these assumptions hold in real CSM interaction. Chatzopoulos et al. verify their model against a more sophisticated hydrodynamical model developed for the H-rich SN 2006gy (Moriya et al. 2013) and obtain a reassuringly similar light curve for similar parameters. Extending the use of the model to H-poor SLSNe, such as PTF 12dam and iPTF 13dcc, is not trivial, however, owing to the different treatment of the opacity when hydrogen is absent. Nevertheless, models that successfully reproduce the light curves of $\mathrm{H}$-poor SLSNe by using CSM interaction have now been reproduced in radiation-hydrodynamics simulations by Sorokina et al. (2016), although these authors do report discrepancies in some parameters of their models in comparison to those obtained with the Chatzopoulos models for the same SLSNe. The semianalytical CSM interaction models remain a valuable tool, and it is clear that $\mathrm{H}$-poor CSM interaction can reproduce the light curves of 
SLSNe, more naturally explaining features such as premaximum bumps in a self-consistent way. However, these caveats show that caution should be used in interpreting the best-fit model parameters based on $\chi^{2}$ minimization of the semianalytical CSM interaction models.

On a final note, an argument often invoked against the CSM interaction model for hydrogen-poor SLSNe is the lack of narrow emission lines, as observed for Type IIn SNe. However, to date, the spectroscopic signature(s) of interaction with an H-deficient dense CSM has not been investigated through spectral synthesis modeling, due to its complexity.

It is a pleasure to acknowledge the help of Manos Chatzopoulos with the implementation of the semianalytical light-curve models developed by him and his colleagues in our light-curve fitting program. We are grateful to Nir Sapir for enlightening discussions, and to WeiKang Zheng, Kelsey Clubb, and Patrick Kelly for their contribution to the 2013 December 3 Keck/LRIS observations of iPTF 13dcc. We thank the staffs at Palomar and Lick Observatories for their expert assistance. The intermediate Palomar Transient Factory project is a scientific collaboration among the California Institute of Technology, Los Alamos National Laboratory, the University of Wisconsin at Milwaukee, the Oskar Klein Center, the Weizmann Institute of Science, the TANGO Program of the University System of Taiwan, and the Kavli Institute for the Physics and Mathematics of the Universe. This paper is based in part on observations made with the NASA/ESA Hubble Space Telescope. Some of the data presented herein were obtained at the W.M. Keck Observatory, which is operated as a scientific partnership among the California Institute of Technology, the University of California, and NASA; the observatory was made possible by the generous financial support of the W. M. Keck Foundation. Research at Lick Observatory is partially supported by a generous gift from Google. These results also made use of the Discovery Channel Telescope at Lowell Observatory. Lowell is a private, nonprofit institution dedicated to astrophysical research and public appreciation of astronomy and operates the DCT in partnership with Boston University, the University of Maryland, the University of Toledo, Northern Arizona University, and Yale University. LMI construction was supported by a grant AST1005313 from the National Science Foundation.

The Dark Cosmology Centre is funded by the DNRF. A.G.Y. is supported by the EU/FP7 via ERC grant No. 307260, the Quantum Universe I-Core program by the Israeli Committee for planning and funding, and the ISF, GIF, Minerva, and ISF grants, WIS-UK "making connections," and Kimmel and ARCHES awards. Support for D.A.P. was provided by NASA through Hubble Fellowship grant HST-HF-51296.01-A awarded by the Space Telescope Science Institute, which is operated by the Association of Universities for Research in Astronomy, Inc., for NASA, under contract NAS 5-26555. M.S. acknowledges support from EU/FP7-ERC grant 615929. E.O.O. is incumbent of the Arye Dissentshik career development chair and is grateful to support by grants from the Willner Family Leadership Institute Ilan Gluzman (Secaucus NJ), Israel Science Foundation, Minerva, and the I-CORE Program of the Planning and Budgeting Committee and The Israel Science Foundation. Support for I.A. was provided by NASA through the Einstein Fellowship Program, grant PF6-170148. A.V.F.'s supernova group at UC Berkeley is supported through NSF grant AST-1211916, the TABASGO Foundation, and the Christopher R. Redlich Fund. The National Energy Research Scientific Computing Center, which is supported by the Office of Science of the U.S. Department of Energy under Contract No. DE-AC02-05CH11231, provided staff, computational resources, and data storage for this project. Part of this research was carried out at the Jet Propulsion Laboratory, California Institute of Technology, under a contract with the National Aeronautics and Space Administration.

\section{REFERENCES}

Ahn, C. P., Alexandroff, R., Allende Prieto, C., et al. 2014, ApJS, 211, 17 Arnett, W. D. 1980, ApJ, 237, 541

Arnett, W. D. 1982, ApJ, 253, 785

Baklanov, P. V., Sorokina, E. I., \& Blinnikov, S. I. 2015, AstL, 41, 95 Barbary, K., Dawson, K. S., Tokita, K., et al. 2009, ApJ, 690, 1358 Benetti, S., Nicholl, M., Cappellaro, E., et al. 2014, MNRAS, 441, 289 Blinnikov, S. I., \& Sorokina, E. I. 2010, arXiv:1009.4353

Brown, T. M., Baliber, N., Bianco, F. B., et al. 2013, PASP, 125, 1031 Cardelli, J. A., Clayton, G. C., \& Mathis, J. S. 1989, ApJ, 345, 245

Cenko, S. B., Fox, D. B., Moon, D.-S., et al. 2006, PASP, 118, 1396 Chatzopoulos, E., Wheeler, J. C., \& Vinko, J. 2012, ApJ, 746, 121

Chatzopoulos, E., Wheeler, J. C., Vinko, J., Horvath, Z. L., \& Nagy, A. 2013, ApJ, 773, 76

Chen, T.-W., Smartt, S. J., Jerkstrand, A., et al. 2015, MNRAS, 452, 1567

Chen, T.-W., Smartt, S. J., Yates, R. M., et al. 2016, arXiv:1605.04925

Chevalier, R. A. 1982, ApJ, 258, 790

Chevalier, R. A., \& Fransson, C. 1994, ApJ, 420, 268

Chevalier, R. A., \& Irwin, C. M. 2011, ApJL, 729, L6

Chugai, N. N., \& Danziger, I. J. 1994, MNRAS, 268, 173

Dessart, L., Hillier, D. J., Waldman, R., Livne, E., \& Blondin, S. 2012, MNRAS, 426, L76

Dessart, L., Waldman, R., Livne, E., Hillier, D. J., \& Blondin, S. 2013 , MNRAS, 428, 3227

Dexter, J., \& Kasen, D. 2013, ApJ, 772, 30

Drake, A. J., Djorgovski, S. G., Mahabal, A., et al. 2009, ApJ, 696, 870

Drake, A. J., Djorgovski, S. G., Mahabal, A. A., et al. 2013, ATel, 5437, 1 Filippenko, A. V. 1997, ARA\&A, 35, 309

Firth, R. E., Sullivan, M., Gal-Yam, A., et al. 2015, MNRAS, 446, 3895 Gal-Yam, A. 2012, Sci, 337, 927

Gal-Yam, A., Mazzali, P., Ofek, E. O., et al. 2009, Natur, 462, 624

Gehrels, N., Chincarini, G., Giommi, P., et al. 2004, ApJ, 611, 1005

Hogg, D. W., Baldry, I. K., Blanton, M. R., \& Eisenstein, D. J. 2002, arXiv: astro-ph/0210394

Hogg, D. W., Blanton, M., Lang, D., Mierle, K., \& Roweis, S. 2008, in ASP Conf. Ser. 394, Astronomical Data Analysis Software and Systems XVII, ed. R. W. Argyle, P. S. Bunclark, \& J. R. Lewis (San Francisco, CA: ASP), 27

Howell, D. A., Kasen, D., Lidman, C., et al. 2013, ApJ, 779, 98

Inserra, C., Smartt, S. J., Gall, E. E. E., et al. 2016, arXiv:1604.01226

Inserra, C., Smartt, S. J., Jerkstrand, A., et al. 2013, ApJ, 770, 128

Japelj, J., Vergani, S. D., Salvaterra, R., Hunt, L. K., \& Mannucci, F. 2016, A\&A, 593, A115

Jerkstrand, A., Smartt, S. J., \& Heger, A. 2016, MNRAS, 455, 3207

Kasen, D., \& Bildsten, L. 2010, ApJ, 717, 245

Kasen, D., Metzger, B. D., \& Bildsten, L. 2016, ApJ, 821, 36

Kasen, D., \& Woosley, S. E. 2007, ApJ, 656, 661

Laher, R. R., Surace, J., Grillmair, C. J., et al. 2014, PASP, 126, 674

Law, N. M., Kulkarni, S. R., Dekany, R. G., et al. 2009, PASP, 121, 1395

Leloudas, G., Chatzopoulos, E., Dilday, B., et al. 2012, A\&A, 541, A129

Leloudas, G., Schulze, S., Krühler, T., et al. 2015, MNRAS, 449, 917

Lunnan, R., Chornock, R., Berger, E., et al. 2014, ApJ, 787, 138

Lunnan, R., Chornock, R., Berger, E., et al. 2015, ApJ, 804, 90

Markwardt, C. B. 2009, in ASP Conf. Ser. 411, Astronomical Data Analysis Software and Systems XVIII, ed. D. A. Bohlender, D. Durand, \& P. Dowler (San Francisco, CA: ASP), 251

Mazzali, P. A., Sullivan, M., Pian, E., Greiner, J., \& Kann, D. A. 2016 , MNRAS, 458, 3455

Miller, J. S., \& Stone, R. P. S. 1993, Lick Obs. Tech. Rep. 66 (Santa Cruz: Univ. California)

Moré, J. J. 1978, in Numerical Analysis, Vol. 630 ed. G. A. Watson (Berlin: Springer), 105 
Moriya, T., Tominaga, N., Tanaka, M., Maeda, K., \& Nomoto, K. 2010, ApJL, 717, L83

Moriya, T. J., Blinnikov, S. I., Tominaga, N., et al. 2013, MNRAS, 428, 1020 Moriya, T. J., Liu, Z.-W., Mackey, J., Chen, T.-W., \& Langer, N. 2015, A\&A, $584, \mathrm{~L} 5$

Moriya, T. J., \& Maeda, K. 2012, ApJL, 756, L22

Moriya, T. J., \& Tominaga, N. 2012, ApJ, 747, 118

Nakar, E., \& Piro, A. L. 2014, ApJ, 788, 193

Neill, J. D., Sullivan, M., Gal-Yam, A., et al. 2011, ApJ, 727, 15

Nicholl, M., Berger, E., Smartt, S. J., et al. 2016, ApJ, 826, 39

Nicholl, M., \& Smartt, S. J. 2016, MNRAS, 457, L79

Nicholl, M., Smartt, S. J., Jerkstrand, A., et al. 2013, Natur, 502, 346

Nicholl, M., Smartt, S. J., Jerkstrand, A., et al. 2015, ApJL, 807, L18

Ofek, E. O., Arcavi, I., Tal, D., et al. 2014, ApJ, 788, 154

Ofek, E. O., Cameron, P. B., Kasliwal, M. M., et al. 2007, ApJL, 659, L13

Ofek, E. O., Laher, R., Law, N., et al. 2012, PASP, 124, 62

Oke, J. B., Cohen, J. G., Carr, M., et al. 1995, PASP, 107, 375

Oke, J. B., \& Gunn, J. E. 1983, ApJ, 266, 713

Pastorello, A., Smartt, S. J., Botticella, M. T., et al. 2010, ApJL, 724, L16

Perley, D. A., Quimby, R. M., Yan, L., et al. 2016, ApJ, 830, 13

Pinto, P. A., \& Eastman, R. G. 2000, ApJ, 530, 757

Piro, A. L. 2015, ApJL, 808, L51

Planck Collaboration, Ade, P. A. R., Aghanim, N., et al. 2016, A\&A, 594, A13

Quimby, R. M., Arcavi, I., Sternberg, A., et al. 2012, ATel, 4121, 1
Quimby, R. M., Kulkarni, S. R., Kasliwal, M. M., et al. 2011, Natur, 474, 487 Rabinak, I., \& Waxman, E. 2011, ApJ, 728, 63

Rahmer, G., Smith, R., Velur, V., et al. 2008, Proc. SPIE, 7014, 70144Y

Rau, A., Kulkarni, S. R., Law, N. M., et al. 2009, PASP, 121, 1334

Sapir, N., \& Waxman, E. 2016, arXiv: 1607.03700

Schlafly, E. F., \& Finkbeiner, D. P. 2011, ApJ, 737, 103

Smith, M., Sullivan, M., D’Andrea, C. B., et al. 2016, ApJL, 818, L8

Smith, N., Li, W., Foley, R. J., et al. 2007, ApJ, 666, 1116

Sorokina, E., Blinnikov, S., Nomoto, K., Quimby, R., \& Tolstov, A. 2016, ApJ, 829, 17

Taddia, F., Fremling, C., Sollerman, J., et al. 2016, A\&A, 592, A89

Thöne, C. C., de Ugarte Postigo, A., García-Benito, R., et al. 2015, MNRAS, 451, L65

Valenti, S., Howell, D. A., Stritzinger, M. D., et al. 2016, MNRAS, 459, 3939

Vreeswijk, P. M., Savaglio, S., Gal-Yam, A., et al. 2014, ApJ, 797, 24

Wang, S. Q., Liu, L. D., Dai, Z. G., Wang, L. J., \& Wu, X. F. 2016, ApJ, 828,87

Wang, S. Q., Wang, L. J., Dai, Z. G., \& Wu, X. F. 2015, ApJ, 799, 107

Wheeler, J. C., Johnson, V., \& Clocchiatti, A. 2015, MNRAS, 450, 1295

Woosley, S. E. 2010, ApJL, 719, L204

Yan, L., Quimby, R., Ofek, E., et al. 2015, ApJ, 814, 108

Yaron, O., \& Gal-Yam, A. 2012, PASP, 124, 668

Young, D. R., Smartt, S. J., Valenti, S., et al. 2010, A\&A, 512, A70

Zacharias, N., Monet, D. G., Levine, S. E., et al. 2004, BAAS, 36, 1418 